\title{
Nuclear Logging and
}

\section{Geothermal Log Interpretation:}

\section{Formation Temperature Sonde Evaluation}

\author{
Edward W. Ross* \\ Nichols Vagelatos* \\ John M. Dickerson* \\ Van Nguyen*
}

\section{NOTICE}

PORTIONC AE T'.' OFPORT ARE ILLEGIBLE. It

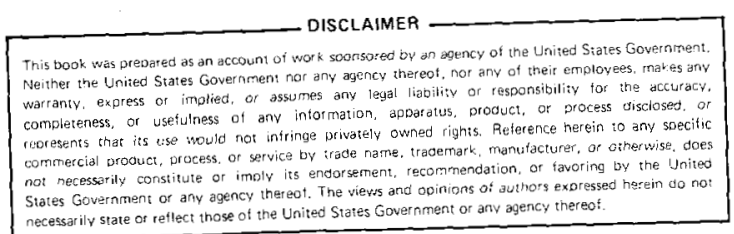
has heen reproduced frem the best available
copy to permit the broadest possible avall ability.

\footnotetext{
*Instrumentation Research Technology Corporation, 7650 Convoy Court, P. O. Box 80817, San Diego, CA 92138.
}

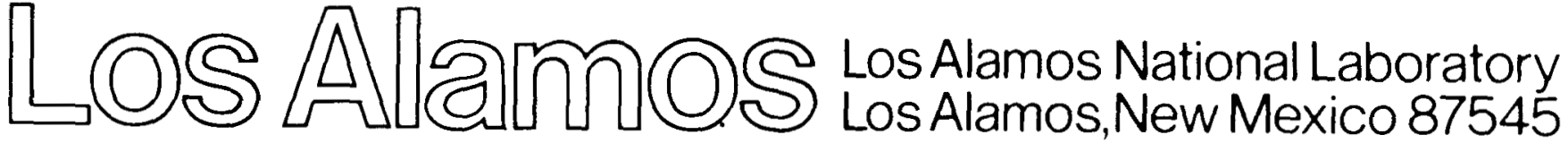




\section{DISCLAIMER}

This report was prepared as an account of work sponsored by an agency of the United States Government. Neither the United States Government nor any agency Thereof, nor any of their employees, makes any warranty, express or implied, or assumes any legal liability or responsibility for the accuracy, completeness, or usefulness of any information, apparatus, product, or process disclosed, or represents that its use would not infringe privately owned rights. Reference herein to any specific commercial product, process, or service by trade name, trademark, manufacturer, or otherwise does not necessarily constitute or imply its endorsement, recommendation, or favoring by the United States Government or any agency thereof. The views and opinions of authors expressed herein do not necessarily state or reflect those of the United States Government or any agency thereof. 


\section{DISCLAIMER}

Portions of this document may be illegible in electronic image products. Images are produced from the best available original document. 


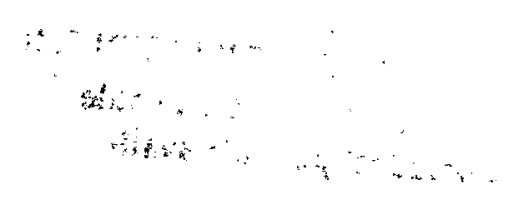




\section{CONTENTS}

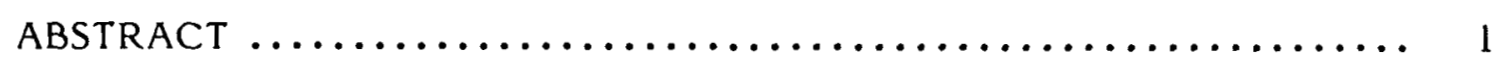

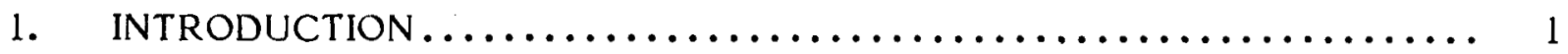

2. TFTS UNDERLYING PRINCIPLES $\ldots \ldots \ldots \ldots \ldots \ldots \ldots \ldots \ldots \ldots \ldots \ldots \ldots \ldots$

2.1 General Theory $\ldots \ldots \ldots \ldots \ldots \ldots \ldots \ldots \ldots \ldots \ldots \ldots \ldots \ldots, \quad 3$

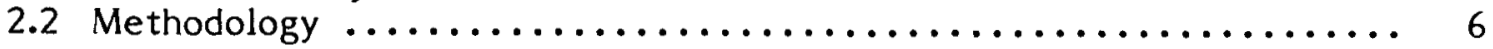

3. HIGH TEMPERATURE TEST STAND DESIGN $\ldots \ldots \ldots \ldots \ldots \ldots \ldots \ldots, 12$

3.1 Borehole Model Description $. . \ldots \ldots \ldots \ldots \ldots \ldots \ldots \ldots \ldots \ldots, 12$

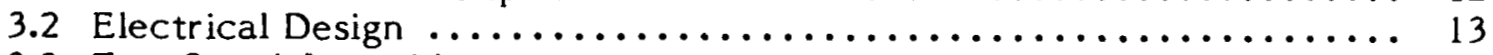

3.3 Test Stand Assembly $\ldots \ldots \ldots \ldots \ldots \ldots \ldots \ldots \ldots \ldots \ldots \ldots \ldots \ldots, 16$

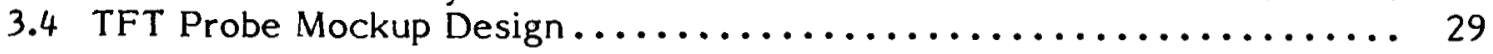

4. TFTS DATA ANALYSIS METHODS $\ldots \ldots \ldots \ldots \ldots \ldots \ldots \ldots \ldots \ldots \ldots \ldots \ldots \ldots \ldots$

4.1 Epither mal Integrals............................... 31

4.2 Evaluation of Thermal Integrals $\ldots \ldots \ldots \ldots \ldots \ldots \ldots \ldots \ldots \ldots \ldots \ldots, 34$

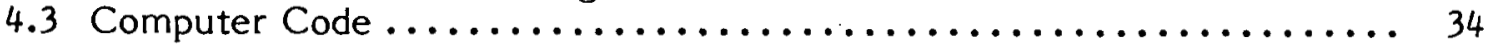

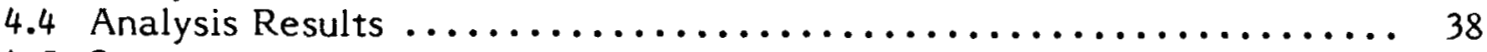

4.5 Summary $\ldots \ldots \ldots \ldots \ldots \ldots \ldots \ldots \ldots \ldots \ldots \ldots \ldots \ldots \ldots \ldots \ldots \ldots \ldots \ldots \ldots \ldots, \quad 40$

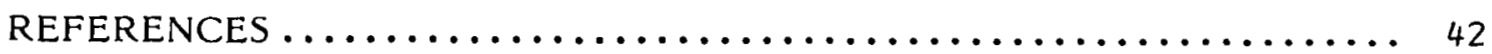

APPENDIX A: RESPONSE OF ${ }^{3}$ He DETECTORS AT HIGH AMBIENT TEMPERATURES $\ldots \ldots \ldots \ldots \ldots \ldots \ldots \ldots \ldots \ldots \ldots, 43$

APPENDIX B: GEOTHERMAL TEST STAND SCHEMATIC (located inside back cover) 


\section{FIGURES}

\section{Figure}

1 Thermal neutron energy distributions in a moderating medium for two different values of the neutron temperature, $T_{n}$, calculated on the

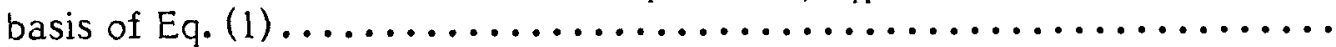

2 Schematic design of the formation temperature sensor showing the essential components of the system.................... 7

3 Data obtained with the sonde mockup $\ldots \ldots \ldots \ldots \ldots \ldots \ldots \ldots \ldots \ldots$

4 Automatic shutdown circuit......................... 13

5 Actuator pump flow $\ldots \ldots \ldots \ldots \ldots \ldots \ldots \ldots \ldots \ldots \ldots \ldots \ldots \ldots \ldots \ldots$

6 Heater banks $\ldots \ldots \ldots \ldots \ldots \ldots \ldots \ldots \ldots \ldots \ldots \ldots \ldots \ldots \ldots \ldots \ldots \ldots$

7 Thermocouples $\ldots \ldots \ldots \ldots \ldots \ldots \ldots \ldots \ldots \ldots \ldots \ldots \ldots \ldots \ldots \ldots \ldots \ldots$

8 Oil level float switch (not to scale) $\ldots \ldots \ldots \ldots \ldots \ldots \ldots \ldots \ldots \ldots \ldots$

9 Test stand during assembly showing tank resting on foam insulator base and concrete slab............................. 18

10 Three 55-gallon drums used as expansion tank for system and overflow pipe.................................... 19

11 Completed tank prior to installing insulation $\ldots \ldots \ldots \ldots \ldots \ldots \ldots$

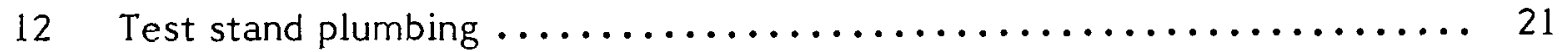

13 Completed system showing insulated tank and plumbing $\ldots \ldots \ldots \ldots \ldots 22$

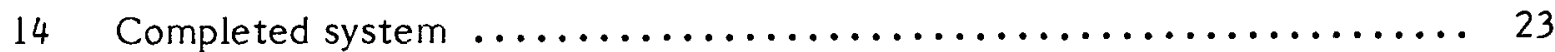

15 Granite stack before assembly $\ldots \ldots \ldots \ldots \ldots \ldots \ldots \ldots \ldots \ldots \ldots \ldots \ldots$

16 Granite slab lifting brackets and borehole-centering tool .......... 25

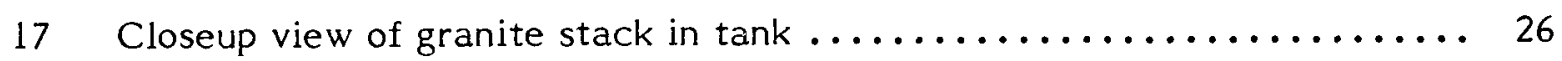

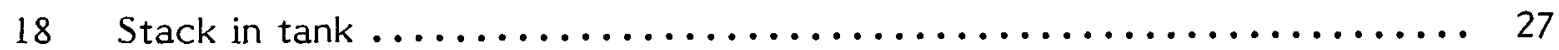

19 True formation temperature probe mockup in test stand ........... 30.

20 Geothermal test stand temperature versus time $\ldots \ldots \ldots \ldots \ldots \ldots$

21 Ratio of Gd-covered detector counts to bare detector counts versus temperature ............................. 40 


\title{
NUCLEAR LOGGING AND GEOTHERMAL LOG INTERPRETATION: FORMATION TEMPERATURE SONDE EVALUATION
}

By

\author{
Edward W. Ross, Nichols Vagelatos, \\ John M. Dickerson, and Van Nguyen
}

\begin{abstract}
The theory and methodology of the neutron-based technique for the determination of the formation temperature in geothermal fields are discussed. The feasibility of the method was demonstrated before start of the present development phase. The present work is intended to evaluate the response of the temperature probe in a simulated fracture porosity granite matrix at temperatures likely to be encountered in known geothermal reservoirs. An above ground borehole model has been designed and constructed. The effect of high ambient temperatures on the response of the neutron detectors in the probe mockup used in the measurements was investigated and used to correct the detector counts. An improved data analysis method has been developed to account properly for the effects of low porosity and high temperatures. Measurements, using the above ground borehole model, have shown that a linear correlation between the ratio of thermal counts from a Gd-filtered detector to counts from a bare detector and formation temperature is good at temperatures as high as $380^{\circ} \mathrm{F}$. The present results are consistent with earlier data obtained in high-porosity laboratory models at lower temperatures $\left(T<167^{\circ} \mathrm{F}\right)$. Further measurements at high temperature at various porosities and formation neutron absorption cross sections would be necessary for a more extensive comparison.
\end{abstract}

\section{INTRODUCTION}

Certain geothermal well or reservoir parameters often cannot be determined reliably by conventional means. Such parameters may be essential in assessing the reservoir potential, or in providing a basis for timely decision making for field development and efficient resource exploitation. Formation temperature is one of these parameters. Conventional means for determining geothermal formation temperature are indirect, extremely time consuming, and often not very reliable. Current techniques make use of conventional temperature sensors, e.g., thermocouples and 
thermistors, to measure the temperature of the borehole fluid. The formation temperature is inferred either by extrapolation of the fluid temperature change with time or by assuming thermal (temperature) equilibrium between the borehole fluid and the surrounding formation. The extrapolation technique requires many hours to several days for a single temperature determination. The alternate method is even slower, requiring many days (even a few months) for the onset of temperature equilibrium. Both methods are adversely affected by fluid circulation.

As a result of the above, a need has existed for an improved technique and instrumentation to determine the formation temperature unambiguously, and also faster and more economically than with the current methods. In response to this need, an advanced neutron-based technique was conceived at Instrumentation Research Technology (IRT) Corporation.* Its development was funded initially by the Energy Research and Development Administration through the San Francisco Operations office and later by the Department of Energy (DOE)/Division of Geothermal Energy through Sandia Laboratories. The early development work demonstrated the scientific and practical (engineering) feasibility of the technique and assessed the response of the temperature sensor under conditions departing from the optimum measurement configuration. This work and the results have been doucumented in detail elsewhere and will. not be discussed here. The experimental work performed before the present development phase was confined to laboratory measurements at temperatures below $90^{\circ} \mathrm{C}\left(194^{\circ} \mathrm{F}\right)$. The work to be described in this report is funded by the Division of Geothermal Energy of the DOE through the Los Alamos National Laboratory.

The work performed in the present development phase consists essentially of preparations for further laboratory evaluation of the true formation temperature sonde (TFTS) at temperatures up to at least $200^{\circ} \mathrm{C}\left(392^{\circ} \mathrm{F}\right)$ in a simulated fractured granite formation. The measurements themselves are part of the present phase. Following the successful completion of the phase, it is anticipated that a probe will be designed and built to demonstrate the feasibility of the method in the field.

The present phase of the work involves the design and construction of an above ground borehole model to simulate a fractured granite formation and to achieve temperatures up to at least $200^{\circ} \mathrm{C}\left(392^{\circ} \mathrm{F}\right)$. The temperature probe mockup designed previously was modified to withstand the higher temperatures. The present work also involves measurements of the effect of high ambient temperatures on the response of the neutron detectors used in the probe mockup. This effect must be taken into * Patent allowed and will be issued shortly. 
account in order to interpret properly the temperature sensor response to changes in conditions inside the formation. Development of an improved method of analyzing the detector responses to determine the formation temperature is also part of the current work. This is necessary because previous work was performed in test formations with much higher porosity and at lower temperatures than those intended for the present measurements. As a result, the previous data analysis methods would not account properly for effects due to these differences. The above tasks were completed and measurements were performed at various temperatures up to $200^{\circ} \mathrm{C}\left(392^{\circ} \mathrm{F}\right)$ at a fracture porosity of approximately $2 \%$ (matrix porosity of approximately $0.6 \%$ ). The data were analyzed to determine the sensor response to the formation temperature.

The theory underlying the neutron-based temperature gauging technique and the methodology developed previously are discussed in Section 2 of this report. More detailed information can be found in the cited references to previous work. Section 3 describes the borehole model and sonde mockup designs. The method developed to analyze the data obtained in the high-temperature borehole model and results of the analysis are described in Section 4. The measurements of the detector response at high ambient temperatures and the results of analysis of these data are presented in the Appendix A.

\section{TFTS UNDERLYING PRINCIPLES}

The concepts underlying the neutron-based technique for the determination of the formation temperature are discussed in this section. This discussion of the theory is followed by a description of the general method implementing those concepts. Further details of the method are presented in Ref. 1, which describes the various components of the temperature sensor.

\subsection{General Theory}

When energetic (fast) neutrons are injected into a moderating medium, they propagate through it, undergoing collisions with the surrounding atoms. As a result, their energies are reduced in elastic and inelastic interactions. Unless they are absorbed during the slowing-down process, neutrons eventually achieve thermal equilibrium with the moderating medium. That is, their kinetic energies are reduced to the range of thermal motions of the various atoms. Thereafter, slow neutrons diffuse through the medium (until they are absorbed or they decay) neither losing nor gaining additional energy, in the average, in subsequent 'collisions.

The slow neutron energy distribution is reflected in the energy dependence of the slow neutron flux. This quantity is the product of the density $n(E)$, of neutrons with. 
kinetic energy $E$, and their velocity $v(E)$. It is the neutron field parameter that, in conjunction with the moderator properties, determines the neutron interaction rates, e.g., scattering, absorption, etc. The slow neutron flux is generally represented accurately by the expression

$$
\Phi(E)=2 \pi n\left(\frac{2}{m_{n}}\right)^{1 / 2} \cdot\left(\pi k T_{n}\right)^{-3 / 2} \cdot E \cdot \exp \left(-E / k T_{n}\right)+C E^{-1}
$$

where $\Phi(E)=$ neutron flux, $n=$ neutron density, $m_{n}=$ neutron mass, $k=$ Boltzmann constant, $\mathrm{E}=$ neutron kinetic energy, $\mathrm{T}_{\mathrm{n}}=$ neutron temperature, and $\mathrm{C}=$ constant.

The first term in Eq. (1) is a Maxwellian function representing the thermal component of the moderated (slow) neutron flux. This is the part of the spectrum that is sensitive to the moderator temperature via the parameter $T_{n}$. This parameter is discussed further in following paragraphs. The second term in Eq. (1) consists of neutrons with energies in the slowing-down region of the slow neutron energy distribution. The constant $\mathrm{C}$, which determines the amplitude (neutron population) of this spectral region, is independent of the moderator temperature and neutron absorption, and is determined entirely by the neutron moderating properties of the medium.

The slow neutron energy distribution is determined, in part, by the temperature of the moderating medium. $T_{n}$, which characterizes the thermal component of the spectrum, is a function of the moderator temperature T. Figure 1 presents two slow neutron spectra calculated on the basis of Eq. (1) for the same medium, but for different values of the neutron temperature. These spectra clearly display the dependence of the thermal region on $T_{n}$. Sirice the spectra shown correspond to the same medium, i.e., the same neutron properties, these distributions also exhibit the spectral dependence on moderator temperature. The figure also displays the insensitivity of the spectrum in the slowing-down region to the temperature of the moderating medium.

If the neutron temperature depended on the medium temperature only, i.e., if $T_{n}=T_{n}(T)$, the temperature $T$ could be determined simply by a single measurement of $\Phi(E)$ at $E=E_{0}$ (or in the region $E_{0} \leqslant E \leqslant E_{0}+\Delta E$ ) within the range of the thermal component of the spectrum. The situation, however, is not as simple as this, because, as noted previously, the slow neutron energy distribution depends also on the neutron properties of the moderating medium. More specifically, 


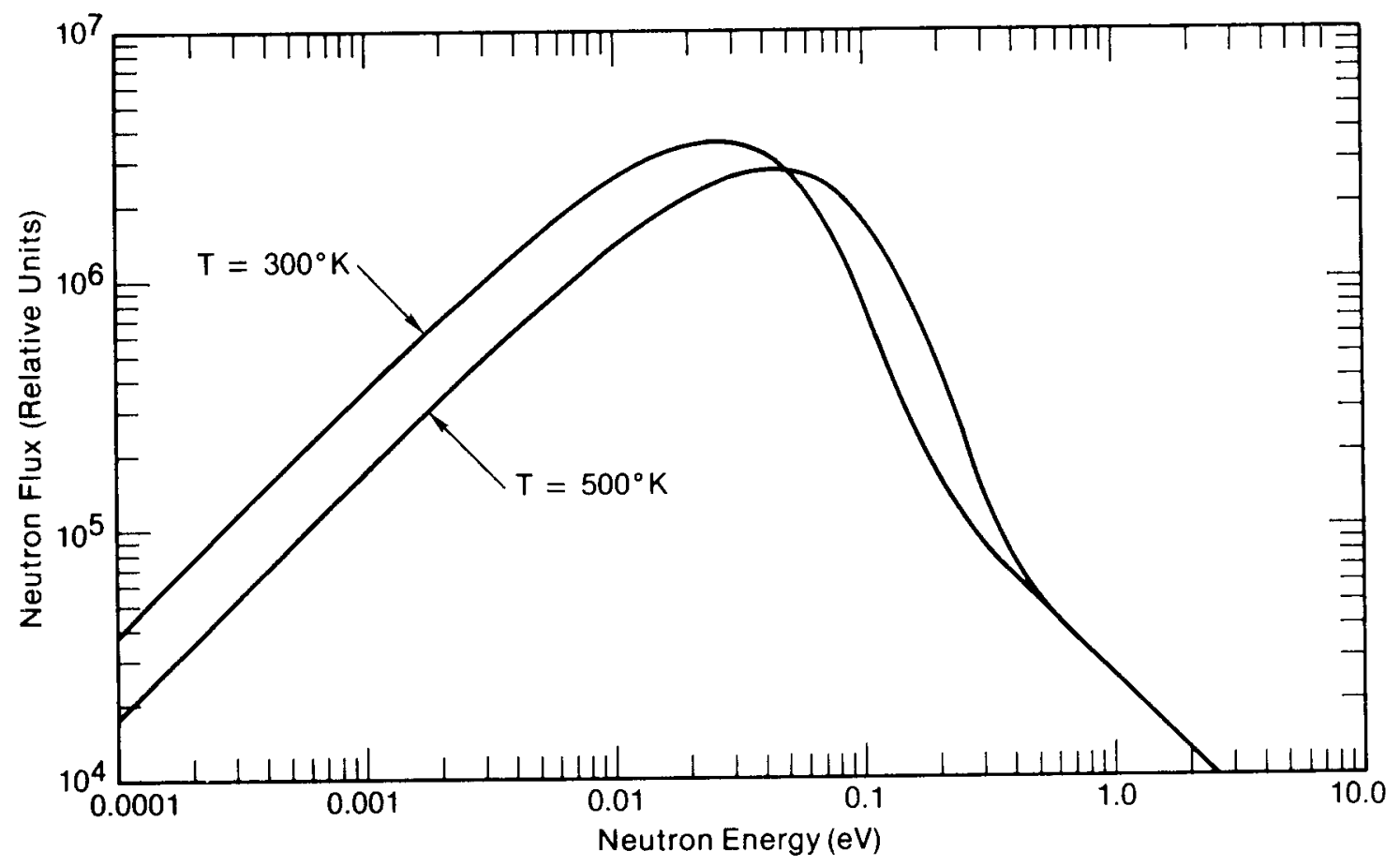

Fig. 1.

Thermal neutron energy distributions in a moderating medium for two different values of the neutron temperature, $T_{n}$, calculated on the basis of Eq. (1). Since the neutron properties of the medium are the same in each case, these distributions clearly display the spectral dependence on the moderator temperature. Generally, $T_{n}=T_{n}\left(T, \Sigma_{a}, \xi \Sigma_{s}\right)$.

$$
\mathrm{T}_{\mathrm{n}}=\mathrm{T}_{\mathrm{n}}\left(\mathrm{T}, \Sigma_{\mathrm{a}}, \xi \Sigma_{\mathrm{s}}\right)
$$

where $\Sigma_{\mathrm{a}} \boldsymbol{\theta}$ neutron absorption cross section, $\Sigma_{\mathrm{s}}=$ neutron scattering cross section, and $\xi=$ average logarithmic energy loss per neutron collision with a moderator atom. $\xi \Sigma_{s}$ is the moderating power of a medium.

The specific functional dependence of $T_{n}$ on the temperature and neutron properties of the moderating medium is determined by the geometry of the medium and the neutron source. For example, in an infinite medium with a uniformly distributed neutron source

$$
\mathrm{T}_{\mathrm{n}}=\mathrm{T} \cdot\left(1+\alpha \frac{\Sigma_{\mathrm{a}}}{\xi \Sigma_{\mathrm{s}}}\right),
$$

where $\alpha$ is an empirical constant. ${ }^{2,3}$ In contrast, the relationship corresponding to the case of a point source in an infinite medium is very complex. In this case, $T_{n}$ is an 
integra! function of $T, \Sigma_{a}$, and $\xi \Sigma_{s^{*}} 1$ Regardless of the complexity of the above expression, however, $T_{n}$ depends on the same three properties of the medium.

Since the information regarding the moderating medium temperature is implicit in the energy distribution of neutrons propagating through it, the temperature can be determined by properly analyzing the moderated neutron spectrum. The implication of the neutron temperature dependence on the neutron properties in addition to temperature is that the procedure is more involved and requires more than the single measurement. It requires the measurement of neutrons with energies in three appropriately chosen regions in the slow neutron energy range. These regions are chosen so that the results of each set of three measurements bears a one-to-one correspondence with the set of conditions under which the measurements are performed, i.e., $\mathrm{T}, \Sigma_{a}$, and $\xi \Sigma_{s}$. This method leads to the unambiguous determination of the medium (formation) temperature and, simultaneously, its neutron absorption cross section and moderating power.

\subsection{Methodology}

Implementation of the above concepts to actually determine the temperature of a geothermal formation involves placing a temperature gauge, in a probe, next to the formation of interest. The temperature sensor consists of a fast neutron source and three detectors sensitive to neutrons with energies in three different regions of the slow neutron range. Figure 2 presents a schematic diagram of the gauge. $\mathrm{A}^{252} \mathrm{Cf}$ fast neutron source and ${ }^{3} \mathrm{He}$ neutron counters are particularly suitable for this application. ${ }^{1}$ The response of the various detectors and the procedure for determining the formation temperature are discussed below with reference to the important general neutron properties of the various components.

The neutron temperature sensor (Fig. 2) is placed downhole next to the formation whose temperature is to be determined. A large fraction of the neutrons emitted by the source enter the formation and propagate through it; a large number of these achieve thermal equilibrium. The slow neutrons diffuse through the formation and many of them eventually escape back into the borehole. The energies of the escaping slow neutrons are distributed in a spectrum identical to that of the slow neutrons still diffusing through the formation. Therefore, the escaping slow neutron spectrum contains all the information regarding the formation temperature and neutron properties. When properly analyzed, this spectrum yields the values of these parameters.

The penetration distance of neutrons into the formation depends on the initial neutron energy and the formation neutron properties. The latter are largely determined by the formation hydrogen content, i.e., saturated porosity, and the salinity of the 
brine. The penetration depth of the formation temperature gauge has been determinec experimentally for a limestone-silica matrix saturated with a boric acid solution. The results of these measurements demonstrate that the penetration depth of the TFTS is similar to that of other neutron-neutron instruments, ${ }^{4-6}$ i.e., 6-12 inches.

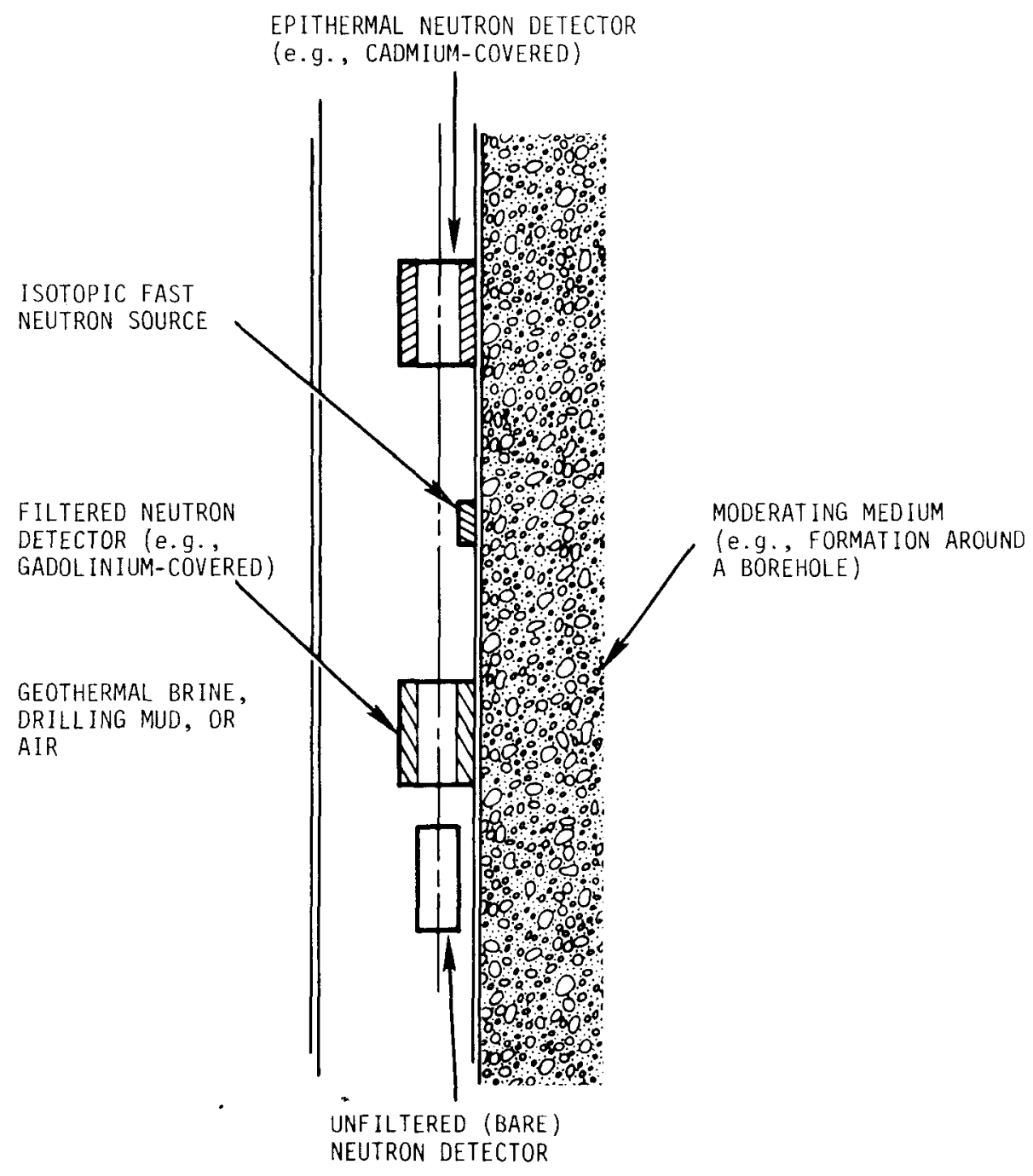

Fig. 2.

Schematic design of the formation temperature sensor showing the essential components of the system. The "sonde" is shown in the side-walled configuration that is appropriate for minimizing borehole fluid interference.

The three neutron detectors are placed in the sonde near the neutron source. When the tool is side walled as in Fig. 2, the detectors are located next to the formation to analyze the escaping slow neutron spectrum with minimum interference from the borehole fluid. The various detectors are made sensitive to neutrons with energies in specific regions of the slow neutron range by covering individual detectors 
with materials having different energy dependent neutron absorption characteristics. Appropriate materials for this application are cadmium (Cd), and gadolinium (Gd). Therefore, one of the three detectors is covered with $\mathrm{Cd}$, the second is covered with Gd, and the third is used bare (unfiltered).

The number of neutrons $\mathrm{C}_{b}$ recorded by a bare (unfiltered) detector exposed to a neutron field is a convolution of the energy dependent neutron flux $\Phi(E)$, at the detector position, and the energy dependent detector counting efficiency $\epsilon(E)$. That is,

$$
C_{b}=\int_{0}^{E_{\max }} \mathrm{dE} \cdot \Phi(E) \cdot \epsilon(E)
$$

where $E_{\text {max }}$ is the maximum kinetic energy of source neutrons. Similarly, the counts recorded by a filtered (gadolinium-, or cadmium-covered) detector $\mathrm{C}_{\mathrm{F}}$ are

$$
C_{F}=\int_{0}^{E_{\max }} d E \cdot \Phi(E) \cdot \epsilon(E) \cdot \tau_{F}\left(E, t_{F}\right)
$$

where $\tau_{F}\left(E, t_{F}\right)$ is the filter transmission for neutrons with energy $E$, and $t_{F}$ is the filter thickness. The filter transmission is determined primarily by the energy-dependent neutron absorption cross section of the filter material. The counts recorded by Gdfiltered and $\mathrm{Cd}$-covered detectors are given by Eq. (5) with the corresponding filter transmission substituted for $\tau_{F}$. Therefore, the Gd-filtered detector counts are

$$
C_{G d}=\int_{0}^{E_{\max }} d E \cdot \Phi(E) \cdot \epsilon(E) \cdot \tau_{G d}\left(E, t_{G d}\right),
$$

where ${ }^{T}{ }_{G d}\left(E, t_{G d}\right)$ is the $G d$ filter transmission function, and $t_{G d}$ is the Gd filter thickness. The Cd-covered detector counts are 


$$
C_{C d}=\int_{0}^{E_{\max }} d E \cdot \Phi(E) \cdot \epsilon(E) \cdot \tau_{C d}\left(E, t_{C d}\right),
$$

where $\tau_{\mathrm{Cd}}\left(\mathrm{E}, \mathrm{t}_{\mathrm{Cd}}\right)$ is the $\mathrm{Cd}$ filter transmission function, and ${ }^{t_{C d}}$ is the $\mathrm{Cd}$ filter thickness.

The $\mathrm{Cd}$ filter transmission is characterized by a sharp cutoff at $0.4 \mathrm{eV}$. Therefore, the cadmium-covered detector is sensitive to neutrons with energies in the epithermal, slowing-down region (Fig. 1) except at very high formation temperatures, i.e., $>1000^{\circ} \mathrm{C}$, or the very low-porosity formations. Since the number of neutrons in this part of the spectrum depends only on the neutron moderating properties of the formation, the response of the Cd-covered detector provides a measure of the moderating power $\xi \Sigma s_{s}$

Similarly, the Gd filter transmission has a rather broad cutoff at energy $0.03 \mathrm{eV}$. Thus, the gadolinium-covered detector is sensitive to neutrons with energies primarily above $\sim 0.03 \mathrm{eV}$ (Fig. 1). This consists of the higher energy portion of the thermal component of the slow neutron spectrum and the slowing-down, epithermal region. Since the fraction of the neutrons in the thermal region accessible to the Gd-filtered detector depends on the neutron temperature $T_{n}$, the response of this detector provides a measure of this parameter.

The bare (unfiltered) detector is sensitive to all slow neutrons with energies in both the thermal and epithermal spectral regions. All else being equal, the number of slow neutrons depends on the absorption cross section of the formation. 7 Therefore, the bare detector response provides a measure of the neutron absorption cross section.

According to the above discussion,

$$
c_{b}=c_{b}^{t h}+c_{b}^{e p}
$$

and

$$
\mathrm{C}_{\mathrm{Gd}}=\mathrm{C}_{\mathrm{Gd}}^{\text {th }}+\mathrm{C}_{\mathrm{Gd}}^{\mathrm{ep}}
$$

where the superscripts "th" and "ep" denote the thermal and epithermal components, respectively. Because 


$$
C_{b}^{e p}=C \cdot \int_{E_{o}}^{E_{\max }} d E \cdot E^{-1} \cdot \epsilon(E)
$$

and

$$
C_{G d}^{e p}=C \cdot \int_{E_{o}}^{E_{\max }} d E \cdot E^{-1} \cdot \epsilon(E) \cdot \tau_{G d}\left(E, t_{G d}\right)
$$

where $E_{o}$ is the low energy cutoff of the slowing-down region (Section 4$), C_{b}^{e p}$ and $C_{G d}^{e p}$ are independent of the formation temperature, and as such they constitute backgrounds for the respective measurements. However, $\mathrm{C}_{\mathrm{b}}^{\mathrm{ep}}$ and $\mathrm{C}_{\mathrm{Gd}}^{\mathrm{ep}}$ can be determined from $\mathrm{C}_{\mathrm{Cd}}^{\text {ep }}$, and $\mathrm{C}_{\mathrm{b}}^{\text {th }}$ and $\mathrm{C}_{\mathrm{Gd}}^{\text {th }}$ can be obtained using Eqs. (8) and (9), respectively.

Since $T_{n}$ is determined by $T, \Sigma_{a}$, and $\xi \Sigma_{s}$, Eq. (2), and $C_{G d}$ th is a measure of the neutron temperature,

$$
\mathrm{C}_{\mathrm{Gd}}^{\mathrm{th}}=\mathrm{f}\left(\mathrm{T}, \Sigma_{\mathrm{a}}, \Sigma_{\mathrm{s}}\right)
$$

or

$$
\mathrm{C}_{\mathrm{Gd}}^{\text {th }} / \mathrm{C}_{\mathrm{b}}^{\mathrm{th}}=\mathrm{g}\left(\mathrm{T}, \Sigma_{\mathrm{a}}, \Sigma_{\mathrm{s}}\right)
$$

and on the basis of the above discussion,

$$
\mathrm{C}_{\mathrm{Gd}}^{\text {th }}=\mathrm{f}^{\prime}\left(\mathrm{T}, \mathrm{C}_{\mathrm{b}}^{\mathrm{th}}, \mathrm{C}_{\mathrm{Cd}}^{\mathrm{ep}}\right)
$$

or

$$
\mathrm{C}_{\mathrm{Gd}}^{\mathrm{th}} / \mathrm{C}_{\mathrm{b}}^{\mathrm{th}}=\mathrm{g}^{\prime}\left(\mathrm{T}, \mathrm{C}_{\mathrm{b}}^{\mathrm{th}}, \mathrm{C}_{\mathrm{Cd}}^{\mathrm{ep}}\right)
$$

The advantage of the ratio $R=C_{G d}^{\text {th }} / C_{b}^{\text {th }}$ is that this quantity is normalized to the same neutron density, Eq. (1). The dependence of the above ratio on the formation temperature is demonstrated by the data presented in Fig. 3. These data were obtained 10 


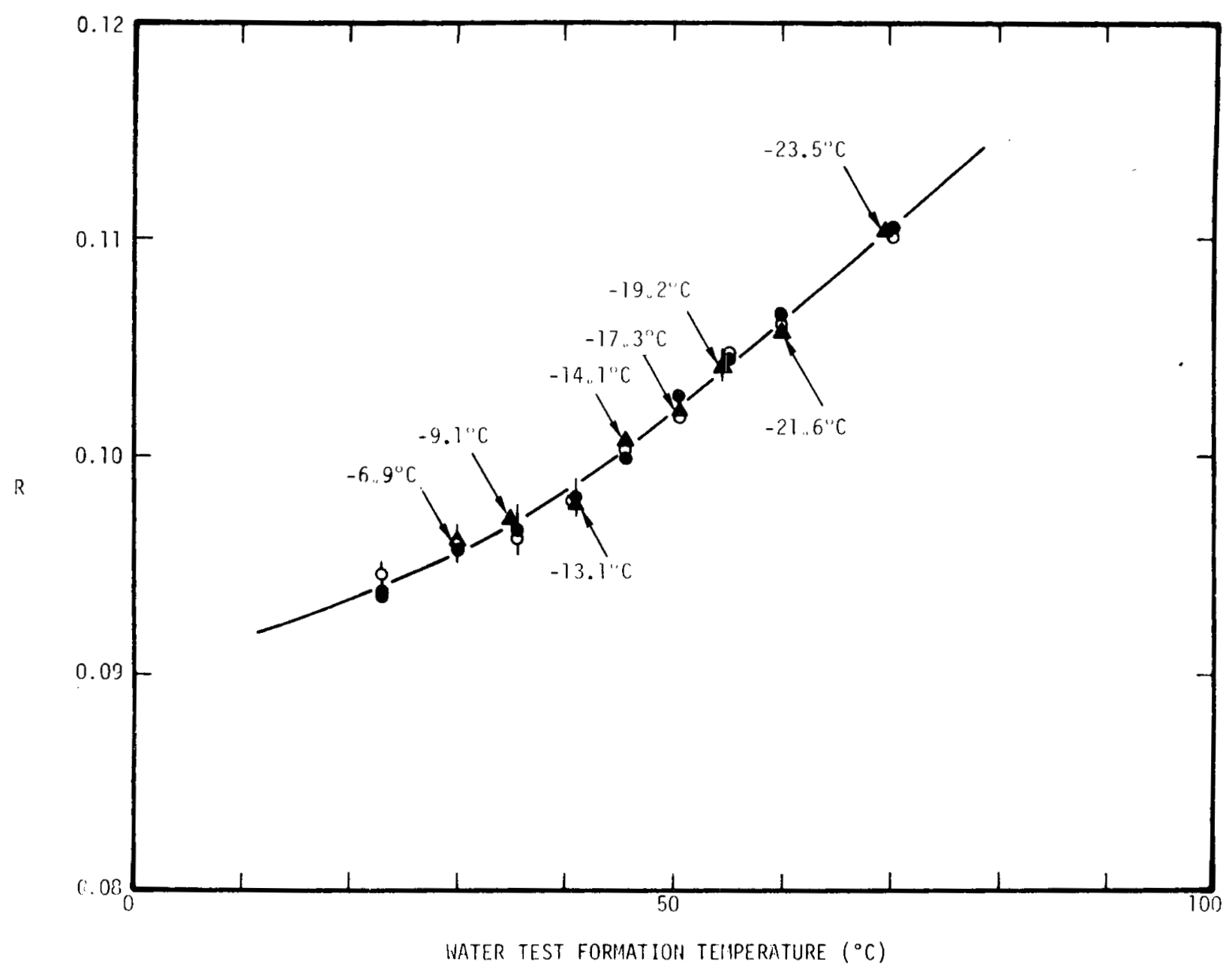

Fig. 3.

Data obtained with the sonde mockup. $R$ is the ratio of the Gd-filtered to bare detector counts after correction for the $1 / \mathrm{E}$ contribution. The test formation is water. Data are shown for the borehole empty (O), the borehole full of water at the temperature of the test formation (O), and at a temperature lower by the number of degrees given $(\Delta)$.

in measurements performed in a borehole model designed to simulate various conditions in a geothermal well. The measurements were among those made to demonstrate the feasibility of the technique. ${ }^{1}$ The proper data analysis method involves a cross plot of the three-detector response. Cross plotting of the corrected detector responses ( $\mathrm{C}_{\mathrm{Gd}}^{\mathrm{t}}$, $\mathrm{C}_{b}^{\text {th }}$, and $\mathrm{C}_{\mathrm{Cd}}^{\mathrm{ep}}$, or $\mathrm{R}, \mathrm{C}_{\mathrm{b}}^{\text {th }}$, and $\mathrm{C}_{\mathrm{Cd}}^{\mathrm{ep}}$ ) along three orthogonal axes constitutes a threedimensional map. Each point on this map determined by the responses of the three detectors corresponds to the specific set of conditions under which these responses were obtained. That is, each point on this mapping corresponds to a unique value of the temperature, $\mathrm{T}$, and each of the neutron properties of the formation, $\Sigma_{\mathrm{a}}$ and $\xi \Sigma \mathrm{s}^{4,6}$ 
Calibration of the TFTS consists of a mapping of the corrected detector responses obtained in well-characterized test formations at accurately known temperatures. The temperature of an arbitrary formation is determined simply by obtaining the threedetector response and locating the corresponding point in the calibration space. The temperature of the formation is the temperature that corresponds to that particular calibration point. Since the correspondence is unique, the temperature determination is unambiguous. Furthermore, the values of the neutron absorption cross sections and the neutron moderating power (formation saturated porosity) are determined simultaneously.

\section{HIGH-TEMPERATURE TEST STAND DESIGN}

\subsection{Borehole Model Description}

The geothermal test stand is a heated-oil device that simulates a fractured granite geothermal environment. The 1200-gallon-capacity main tank contains a hightemperature oil and a stack of 32 granite slabs with a 10-inch borehole in the center. The slabs are 4 feet square and 2 inches thick. They can be stacked one on top of another with spacers between them to obtain the desired porosity. The lowest porosity achievable is the intergranular porosity of the granite used, which is expected to be approximately $0.6 \%{ }^{8}$

The temperature of the simulated formation is varied by heating the oil in the tank. Forty strip heaters are thermally bonded to the outside of the tank and insulated to heat the oil to temperatures up to $200^{\circ} \mathrm{C}\left(392.4^{\circ} \mathrm{F}\right)$.

Thermocouples placed inside the granite stack between the slabs monitor the formation temperature. One of the thermocouples is connected to a temperature controller to maintain the formation temperature at a given set point.

The density of the oil in the tank changes as the temperature changes. The oil level is kept constant by allowing the hot expanding oil to overflow into a 165-gallor. expansion tank consisting of three 55-gallon drums. The oil is also pumped through the tank to prevent the formation of hot spots near the heaters. As the oil cools, the level drops and the oil in the expansion tank is pumped back into the main tank to maintain the oil level above the top of granite stack.

The test stand oil and formation can be heated at a rate of approximately $0.66^{\circ} \mathrm{C}$ $\left(1.2^{\circ} \mathrm{F}\right)$ per hour for the temperature range from $37.8^{\circ} \mathrm{C}\left(100^{\circ} \mathrm{F}\right)$ to $204.4^{\circ} \mathrm{C}\left(400^{\circ} \mathrm{F}\right)$. The oil cools at a rate of approximately $1.1^{\circ} \mathrm{C}\left(2^{\circ} \mathrm{F}\right) /$ hour at temperatures above $65.6^{\circ} \mathrm{C}$ $\left(150^{\circ} \mathrm{F}\right)$. The cooling slows as the temperature approaches room temperature. The 
above rates are based on increasing the temperature in steps of $11.1^{\circ} \mathrm{C}\left(20^{\circ} \mathrm{F}\right)$ and waiting for the oil and granite temperature to equalize.

\subsection{Electrical Design}

Safety features and temperature monitoring are included in the test stand to allow unattended operation of the system. Figures 4 through 7 are electrical diagrams of the geothermal test stand.

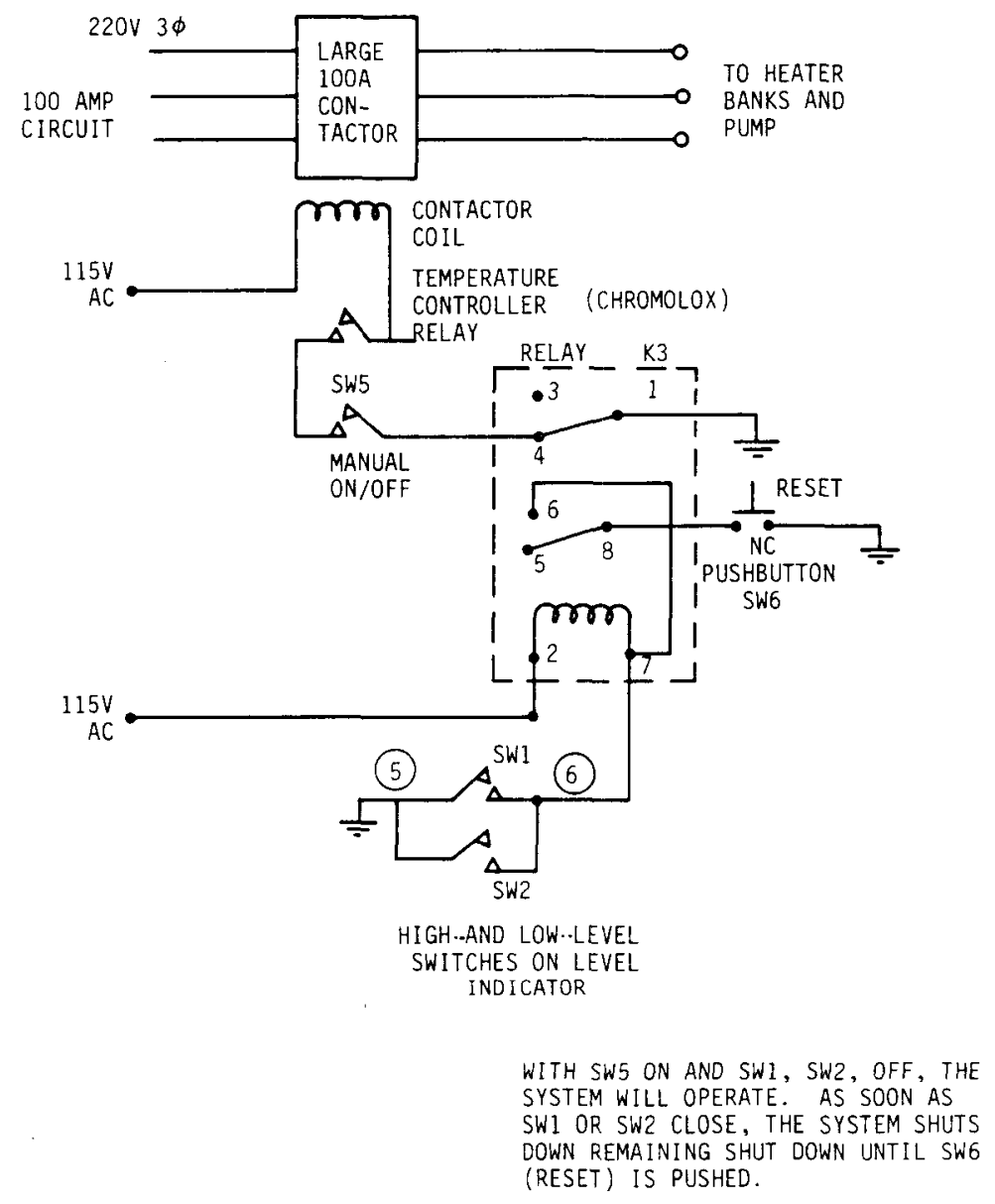

Fig. 4.

Automatic shutdown circuit.

The temperature of the formation is monitored by nine thermocouples placed between the granite slabs. The thermocouple wire is teflon-glass coated, rated for a single measurement to $371^{\circ} \mathrm{C}\left(700^{\circ} \mathrm{F}\right)$. The thermocouples were made by spot welding the wire to a 1 -inch-square sheet of nickel shim stock. The shim stock was then placed 
between two slabs of granite. Table I shows the placement of the thermocouples for this experiment.

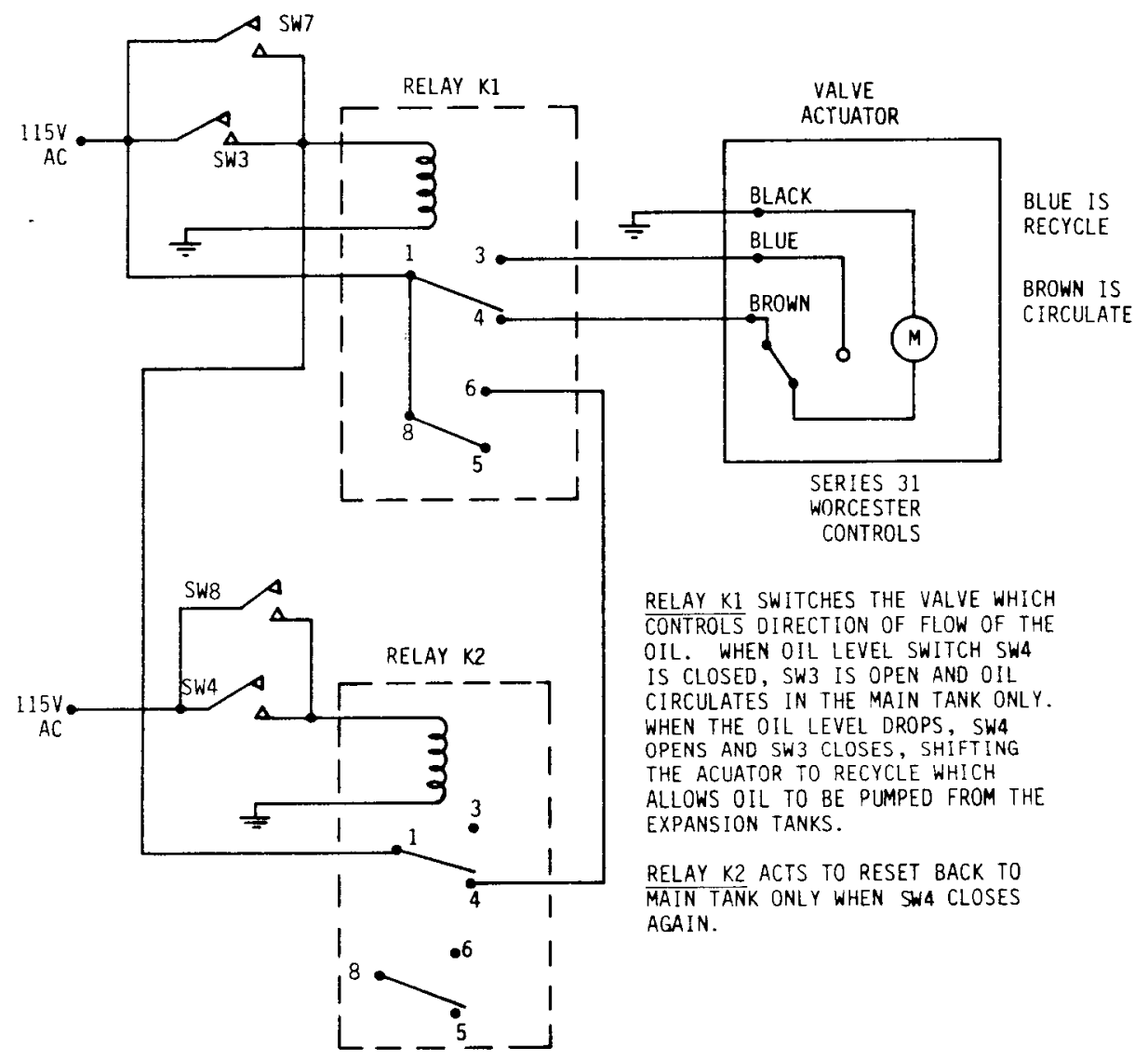

Fig. 5.

Actuator pump flow.

Thermocouple number 5 was connected to the Omega Engineering temperature controller. This controller cycles the bank of strip heaters on and off to maintain the temperature at the setpoint of the controller. A thermocouple was also placed on the surface of one of the strip heaters to TABLE I monitor its temperature as a safety feature. This thermocouple was connected to the Chromalox temperature controller, which was set at a high-temperature cutoff point. If the strip heater's temperature increased above this cutoff point, the Chromalox Controller would shut down, turning off all heaters. The high temperature set point was usually adjusted to be several hundred degrees $C$ above the formation 


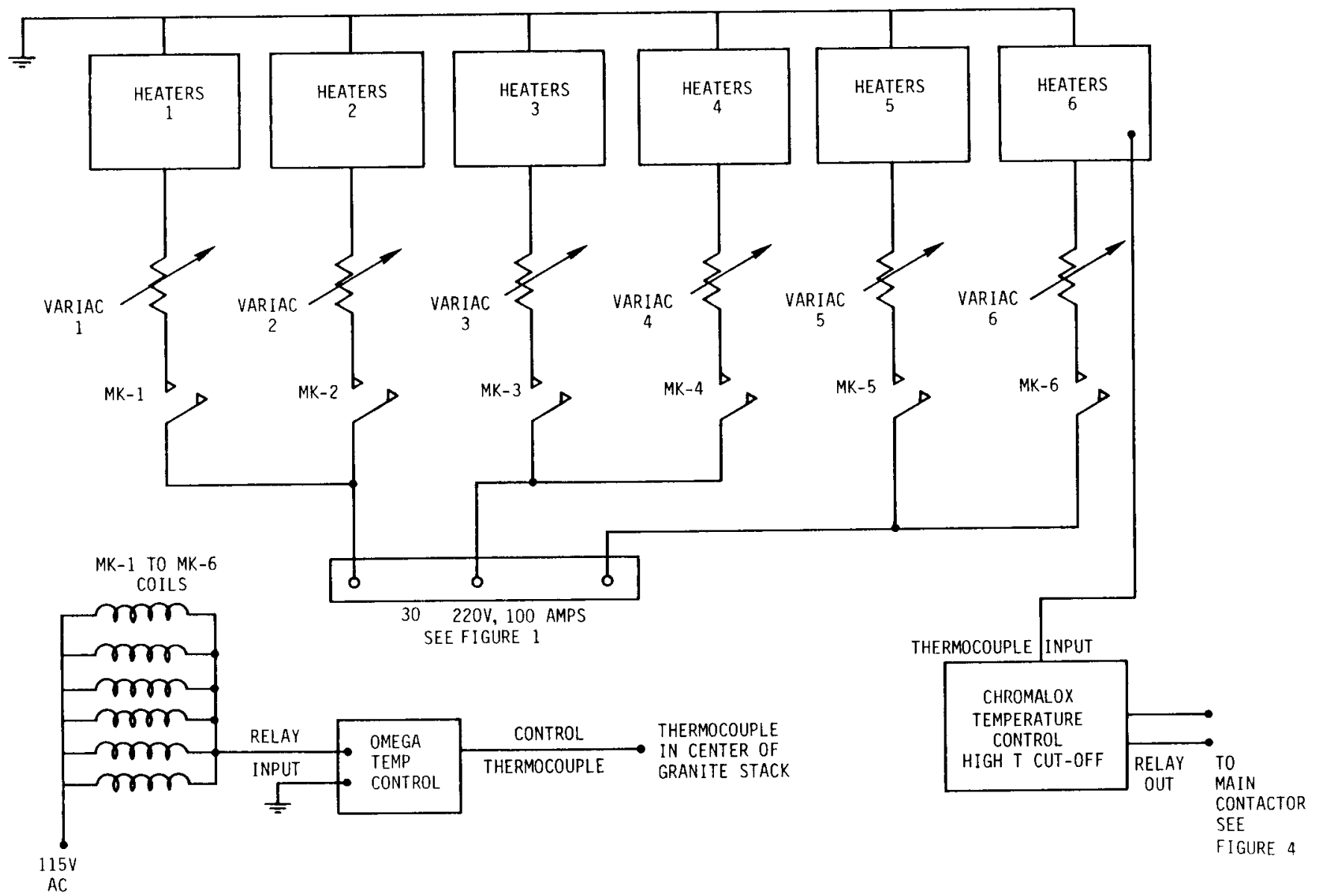

Fig. 6.

Heater banks.

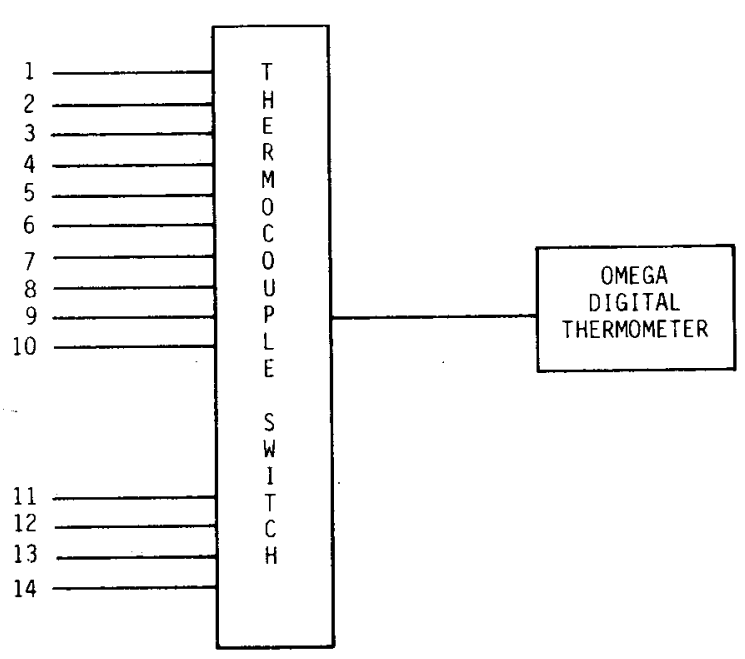

Fig. 7.

Thermocouples. 
temperatures but not higher than $315^{\circ} \mathrm{C}\left(600^{\circ} \mathrm{F}\right.$ ). (The recommended maximum operating temperature to prevent thermal cracking of the oil.) The manufacturer's stated maximum sheath temperature for these heaters is $371^{\circ} \mathrm{C}\left(700^{\circ} \mathrm{F}\right)$.

The strip heaters can also be turned of $f$ by a manual switch. The switch on the oil circulating pump also shuts down the heaters. This is to preclude hot spots in the tank near the heaters when the oil is not circulating.

The oil level is also monitored. Extremely high or low oil levels will cause the entire system to shut down stopping the heaters and pumps. This is accomplished by the float switch shown in Fig. 8. The aluminum bob floats in the oil. As the oil level rises or falls, the magnet on the rod connected to the float travels up and down the tube. Magnetic reed switches at various heights outside the tube are turned on and off as the oil level changes. There are four switches. The top switch closes when the oil level is too high and the system is turned off. The next switch is set at the level of the overflow pipe in the tank. When the switch comes on, an actuator valve turns, stopping the flow of oil out of the expansion tanks. The next lower switch is several inches below the overflow pipe. When this switch closes, theactuator valve is turned to allow pumping of oil out of the expansion tanks into the main tank to raise the oil level. The bottom switch is a safety switch indicating extreme low level. When closed, it shuts down the system.

As shown in Fig. 6 the six banks of strip heaters are connected to $220 \mathrm{~V}$ through variacs to allow variation of heating rates. For each heater bank there is a mercury switch controlled by the temperature controller. The strip heaters are distributed around the outside of the tank at intervals of approximately one foot. Twenty heaters are at the bottom of the tank and the other 20 are near the top.

\subsection{Test Stand Assembly}

The test stand tank was set up on a 7-foot-square concrete slab 3-5/8 inches thick. This provided a flat surface for the tank and granite stack, which weighs approximately $7273 \mathrm{~kg}(16,000 \mathrm{lbs})$.

A layer of foam insulation several inches thick was used on the bottom of the tank. The slab and insulation can be seen in the photograph in Fig. 9, which was taken during assembly of the test stand.

Figure 10 shows the three 55-gallon drums used as an expansion tank for the system and the overflow pipe. Figure 11 shows the completed tank prior to installing the insulation. 


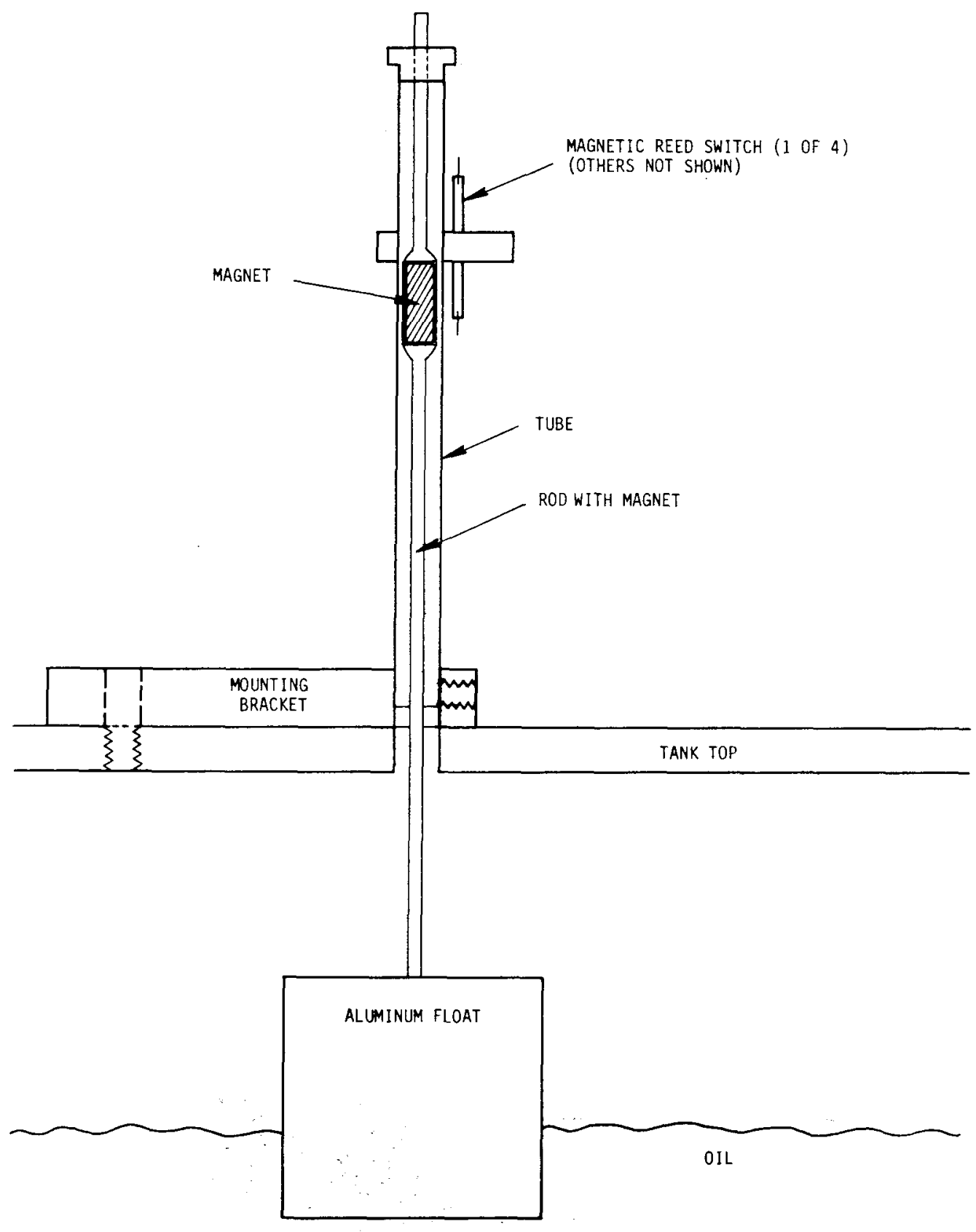

Fig. 8.

Oil level float switch (not to scale).

Figure 12 shows the pump and plumbing for the test stand. The 55-gallon drum in this picture contains Mobiltherm 603 oil used as the heat transfer medium. The flex hose from the 55 -gallon drum leads to the fill/drain pipe for the tank.

The test stand tank and plumbing was insulated to prevent heat loss. Figure 13 shows the complete system. The oil level float switch can be seen in these pictures on 


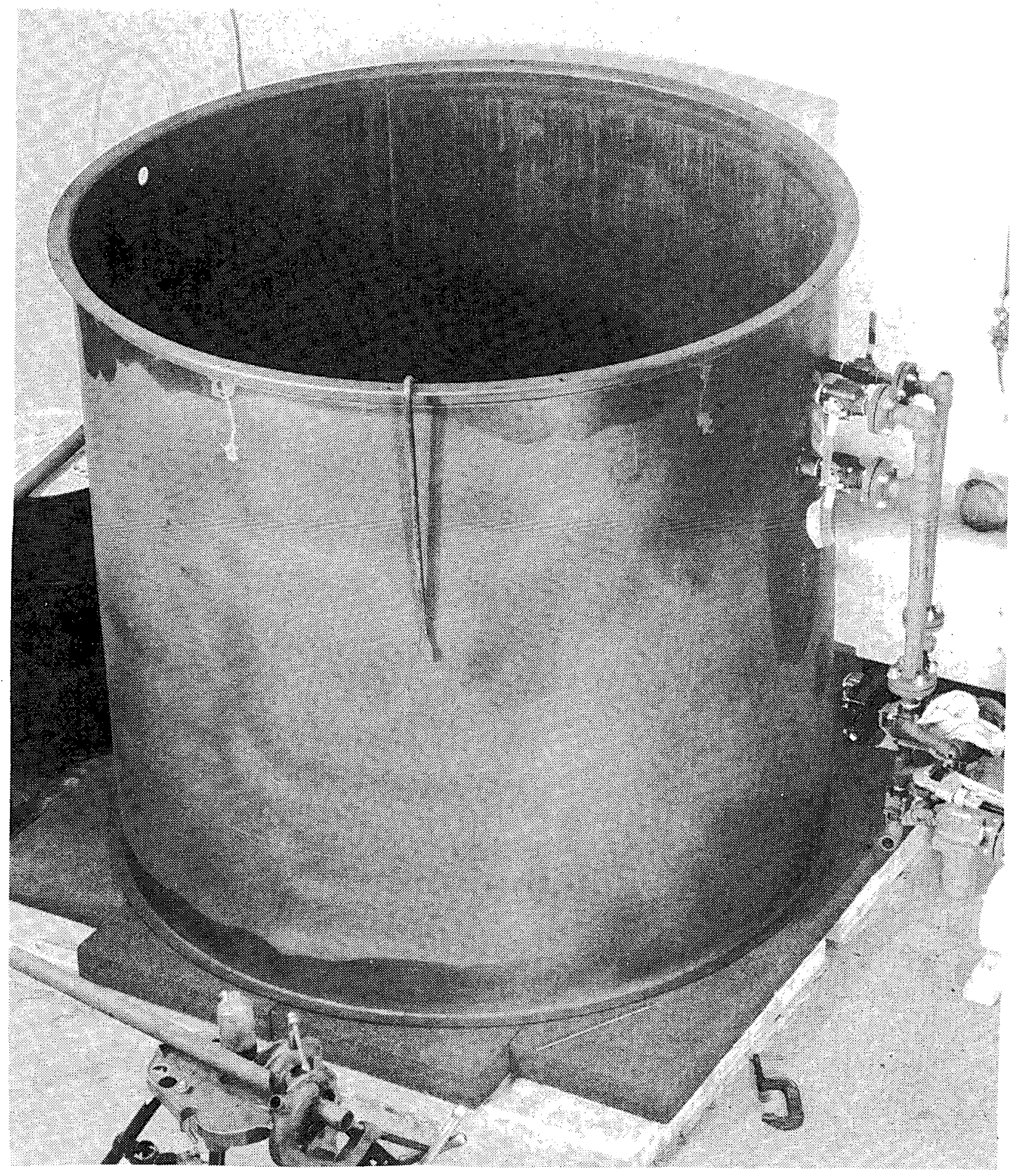

Fig. 9.

Test stand during assembly showing tank resting on foam insulator base and concrete slab. 


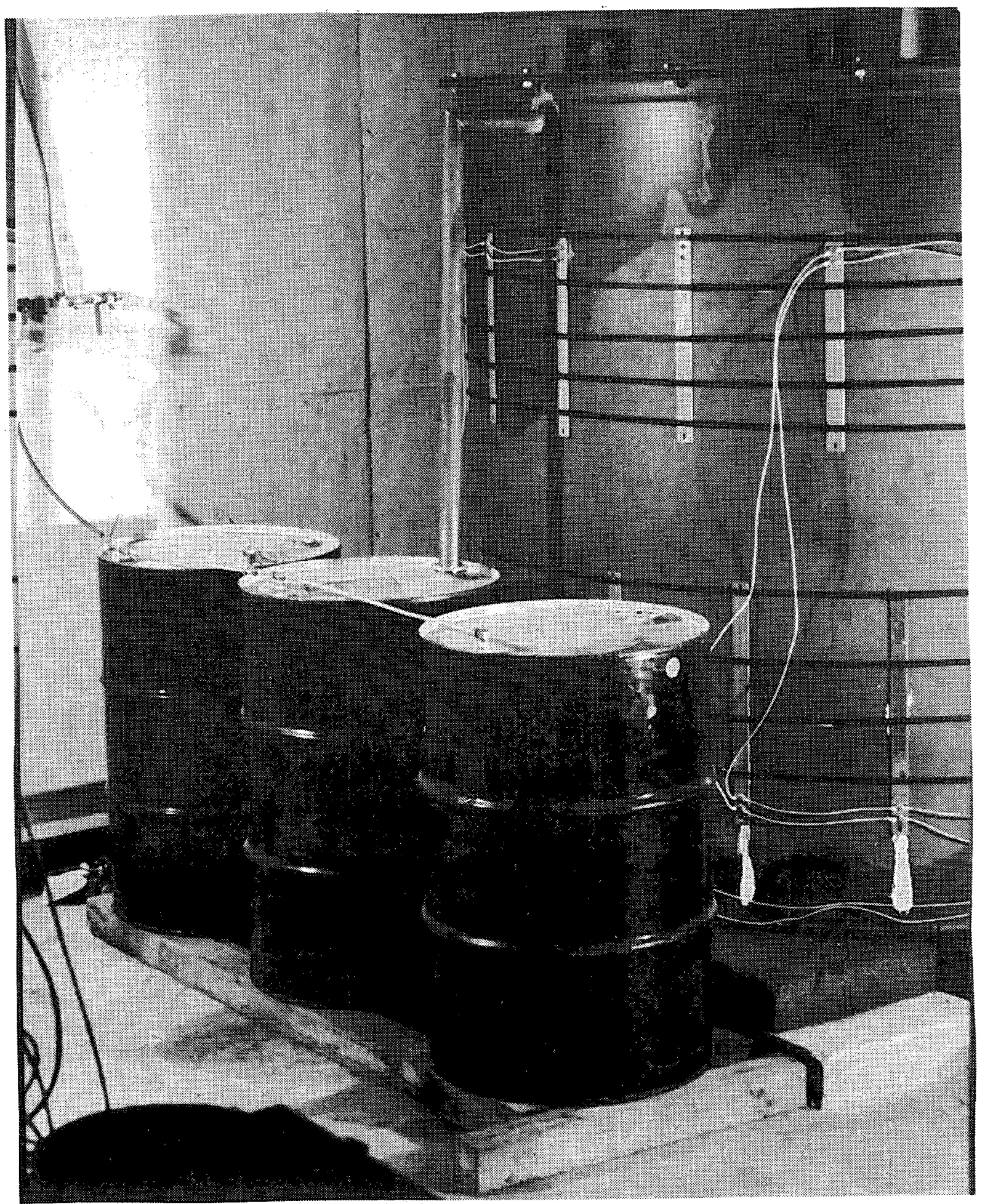

Fig. 10.

Three 55-gallon drums used as expansion tank for system and overflow pipe. 


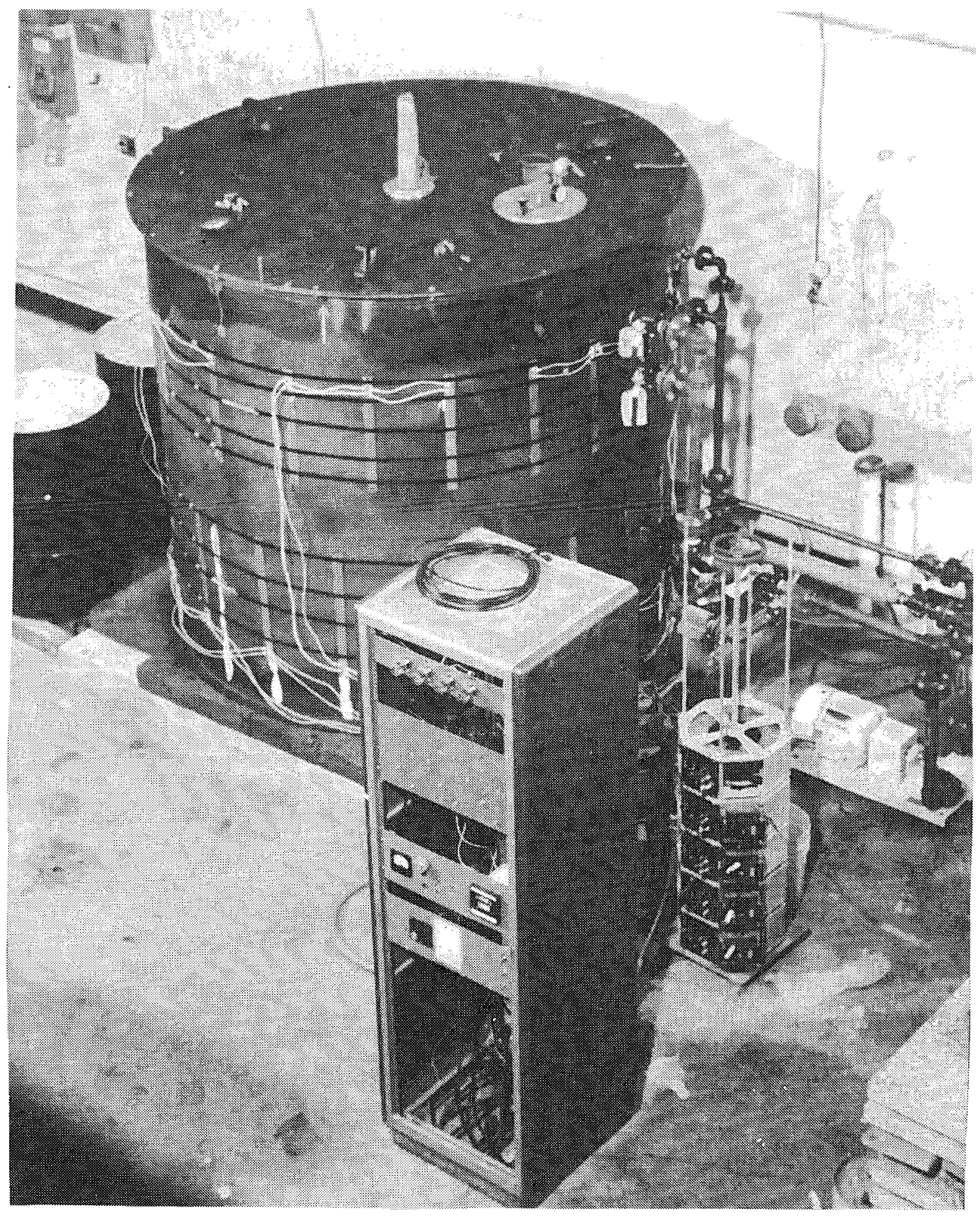

Fig. 11.

Completed tank prior to installing insulation. Shown are banks of heater strips. 


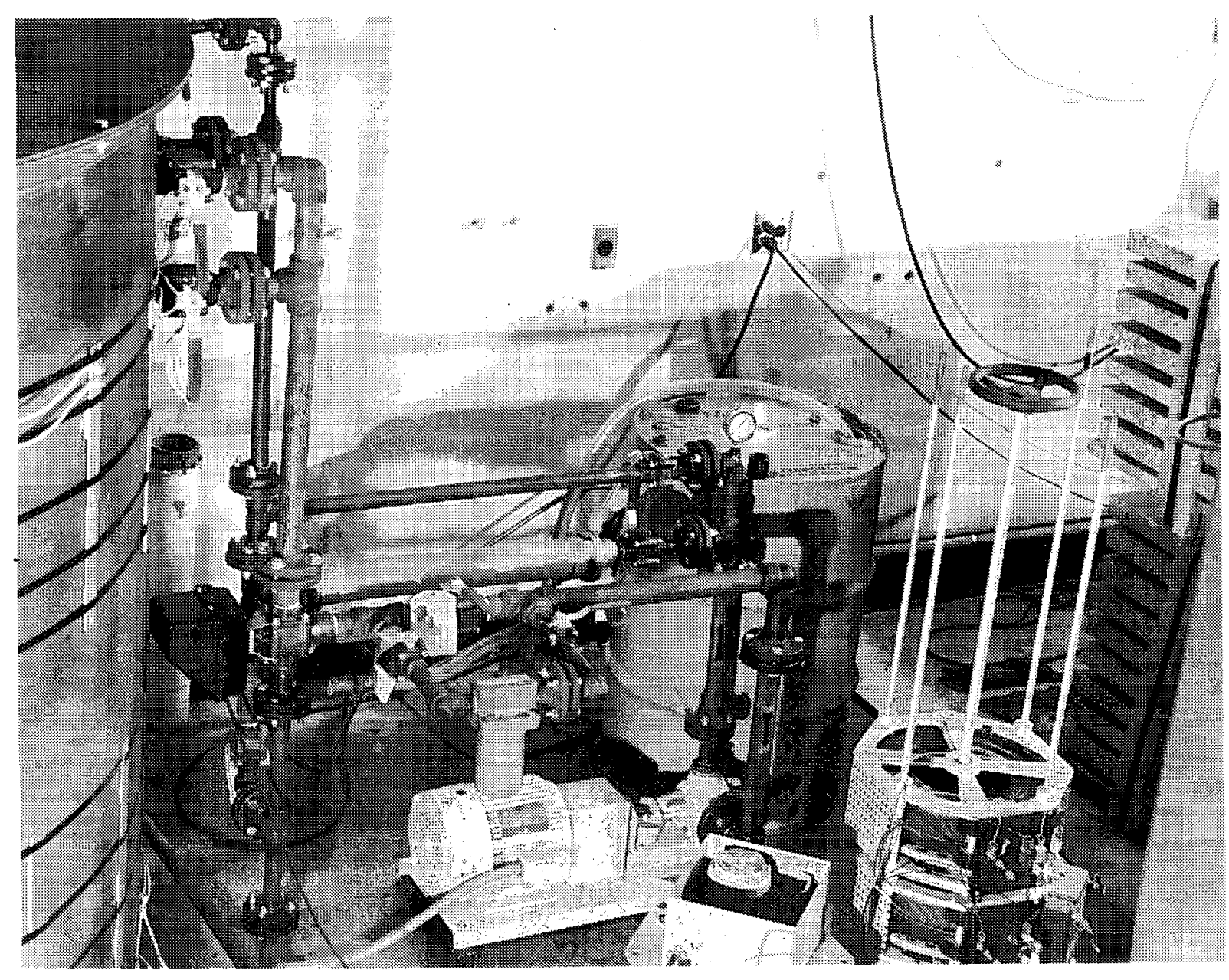

Fig. 12.

Test stand plumbing. 


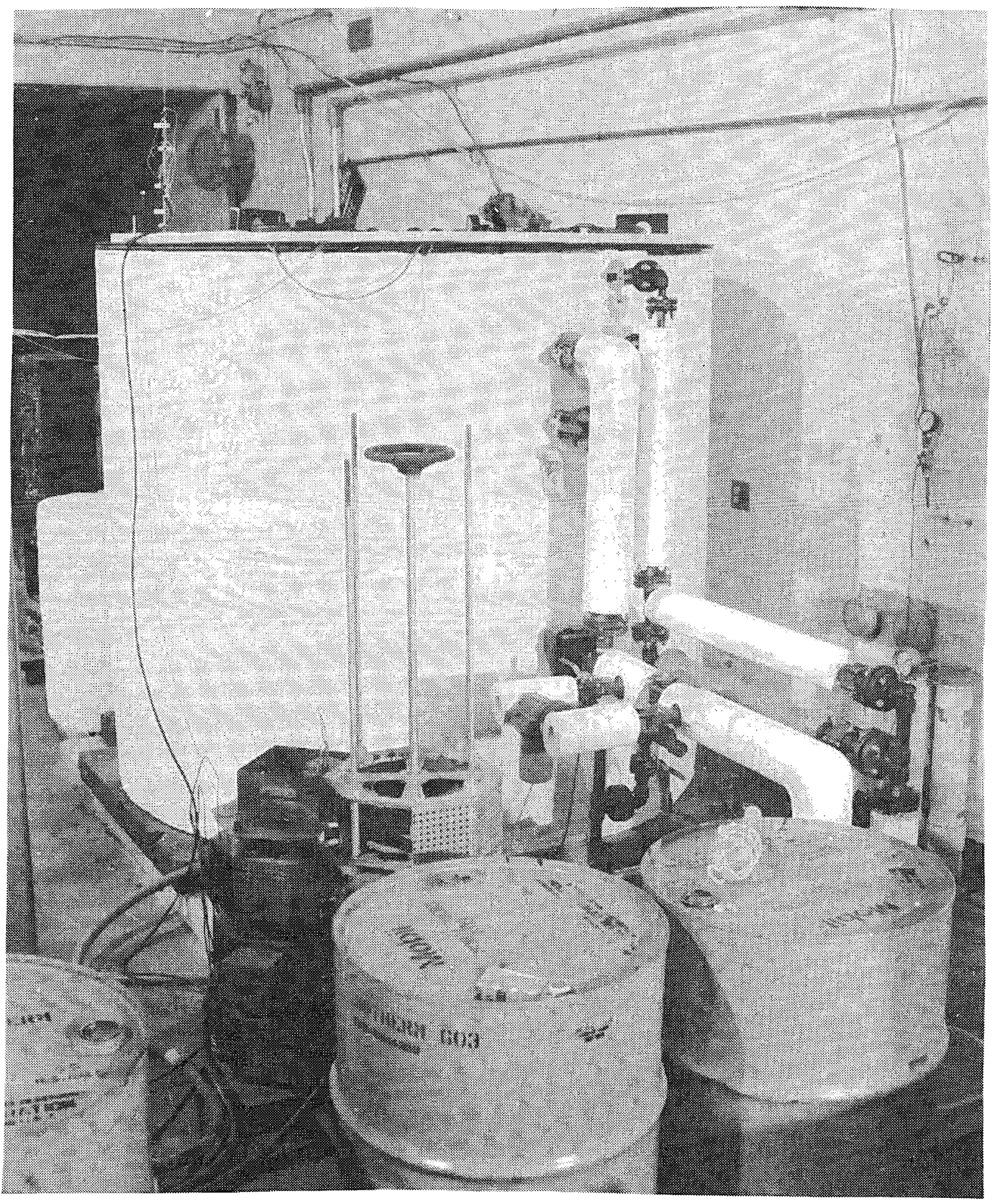

Fig. 13.

Completed system showing insulated tank and plumbing. 
the top of the tank. The relay rack in front of the pump is the test stand control panel containing the relays $\mathrm{K} 1-\mathrm{K} 3$, the mercury relays for the heater banks, and the temperature controllers and gauges. The other relay rack on the left in Fig. 14 contains the nuclear counting electronics.

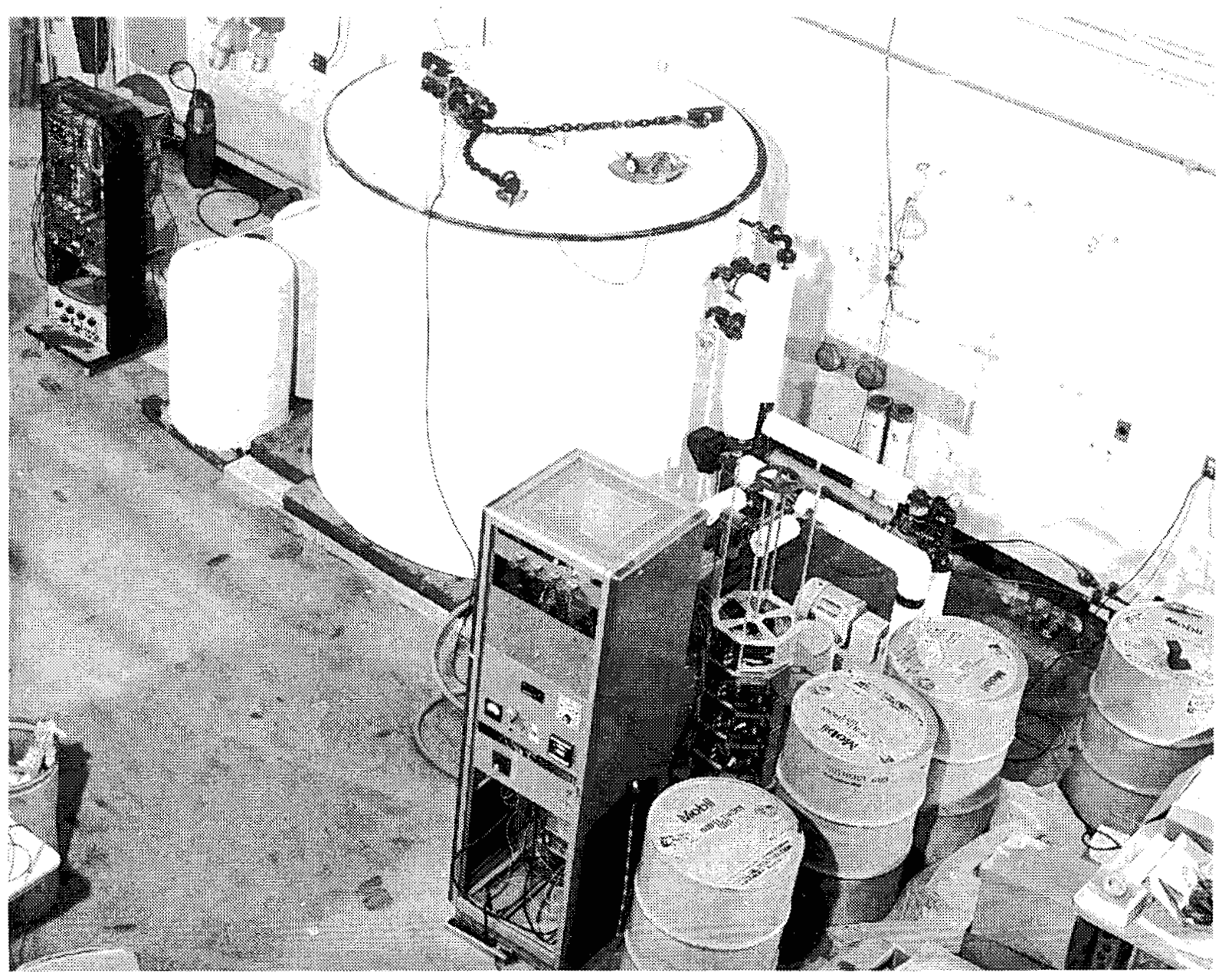

Fig. 14.

Completed system. The equipment rack on left contains nuclear counting electronics.

The granite stack is shown before assembly in Fig. 15. Each slab weighs approximately $227 \mathrm{~kg}$ (500 1bs). The slabs were lifted by an overhead crane into the tank. The slabs were lifted by the corners using the aluminum lifting brackets sketched in Fig. 16. The stack assembly procedure is outlined below.

1. Clean the slab (remove dust and dirt).

2. Lift and position a slab in the tank. The position of the first slab is critical because it must be placed so that the test probe is side walled when the probe 


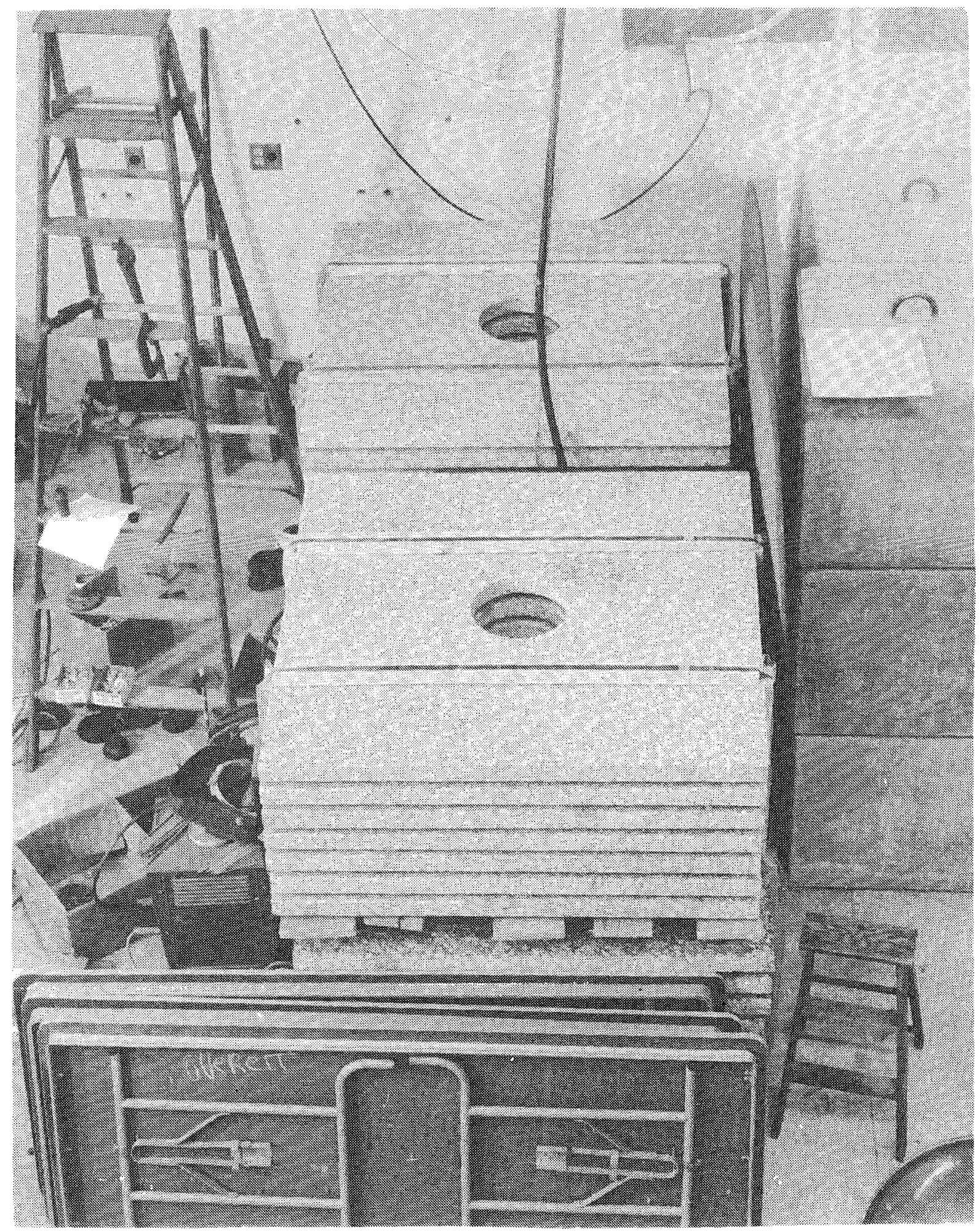

Fig. 15.

Granite stack before assembly. 


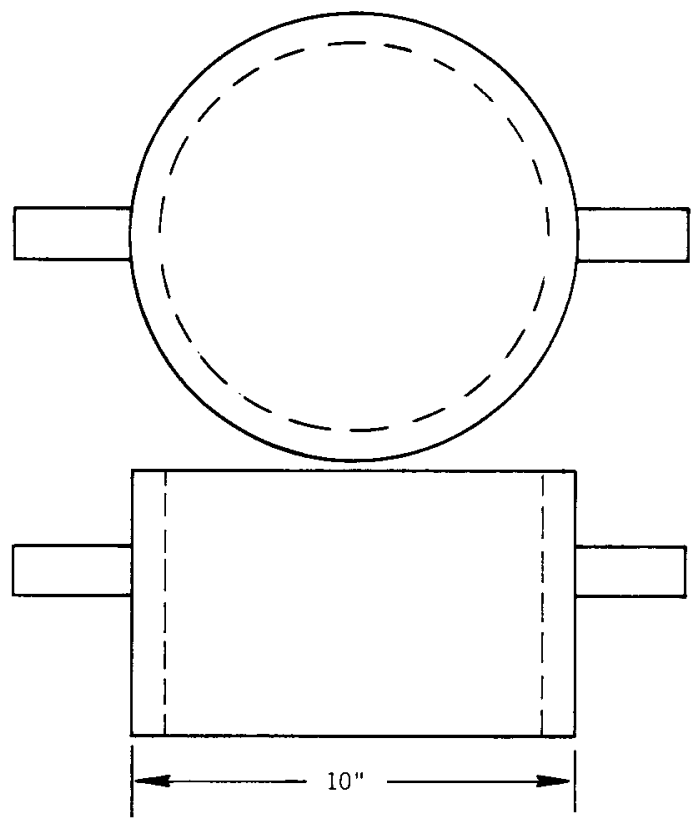

CENTERING TOOL

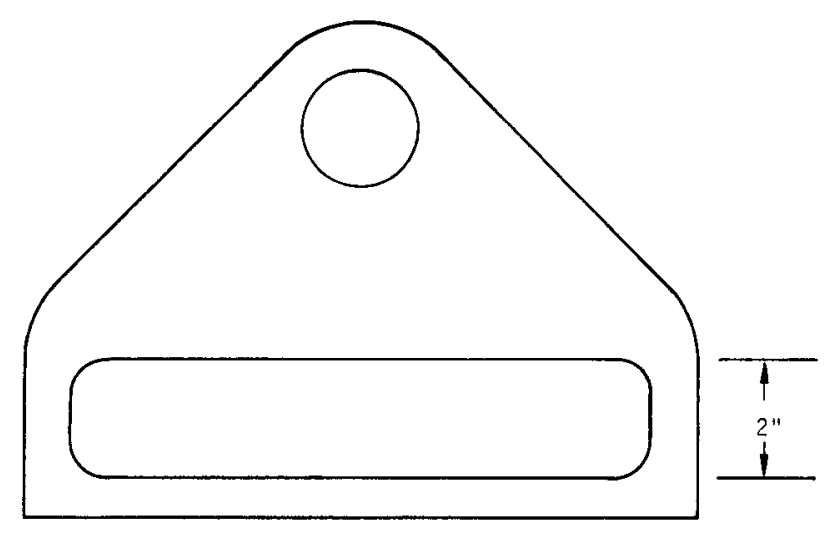

ALUMINUM LIFTING BRACKET

Fig. 16.

Granite slab lifting brackets and borehole-centering tool.

is placed in the test stand. The hole in the slab must align correctly with the hole in the tank top to achieve this. The position of the hole in the tank top for the probe is slightly adjustable, however.

3. Place four spacers on the slab. Spacer thickness determines porosity of the stack. Place thermocouples if desired at this point in the stack. 
4. Lift next slab into tank. Rotate by $45^{\circ}$ to be able to set the slab down. Use the borehole-centering tool to align hole in slab with the hole in the slab below. (Figure 16 shows the centering tool.)

5. Continue with step 2 until all 32 slabs are in place.

Figures 17 and 18 show the completed stack in the test stand.

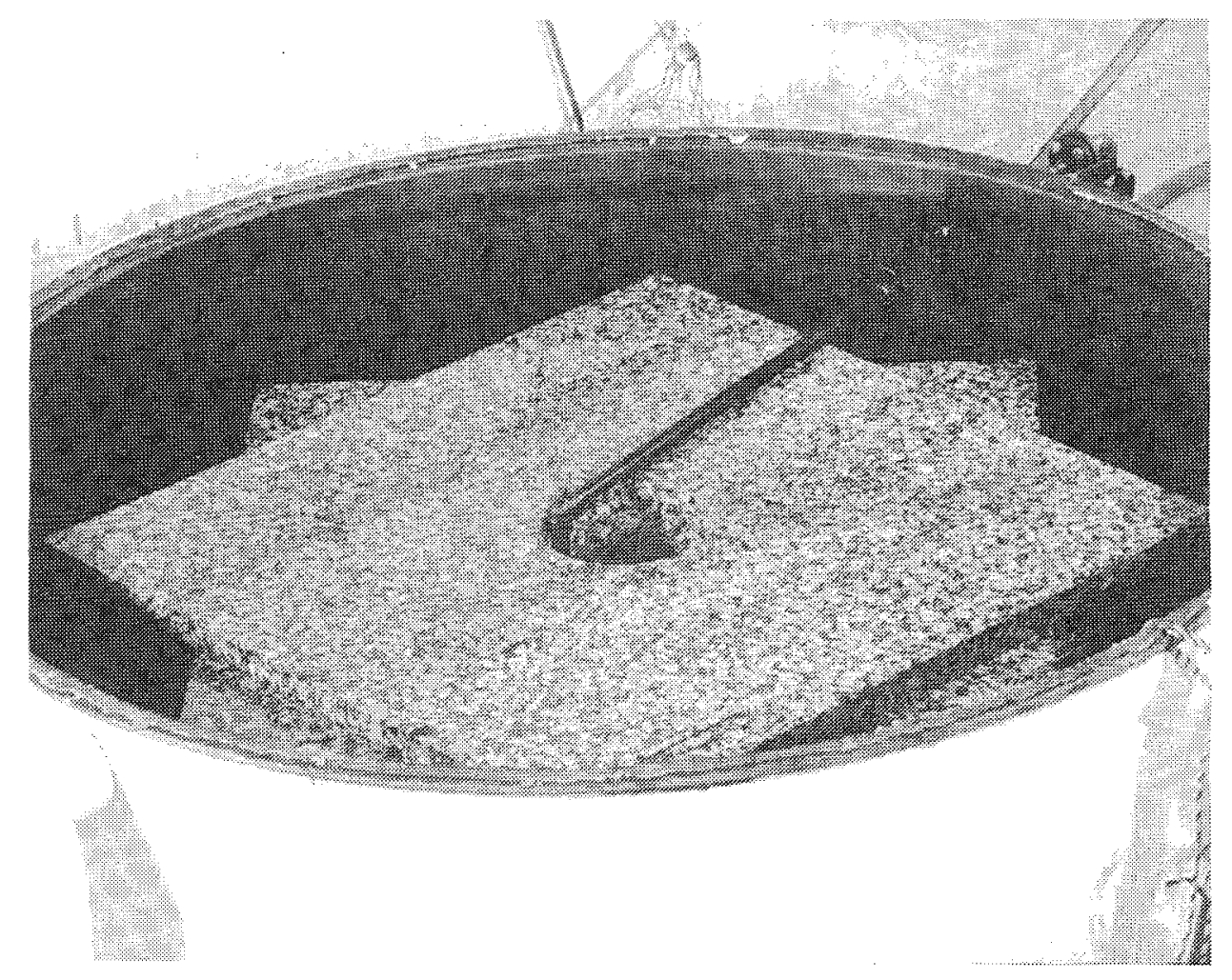

Fig. 17.

Closeup view of granite stack in tank.

After the granite stack is in place, a pipe is connected to direct the oil into the tank from an inlet. Valve 11 controls the flow of oil into the borehole. See the test stand schematic RT122D0001A located in Appendix B in the envelope at the back of this report.

The following procedure was used to fill the tank with oil after the pump was primed. (Refer to RT122D0001A.)

1. Open valve 1 (V1), V9, V10, and V1l.

2. Open V4 and V5; close V3 if filter is to be in cycle.

3. Open V3; close V4 and V5 if no filter.

4. Close V2, V6, V7, V8, and V12. 


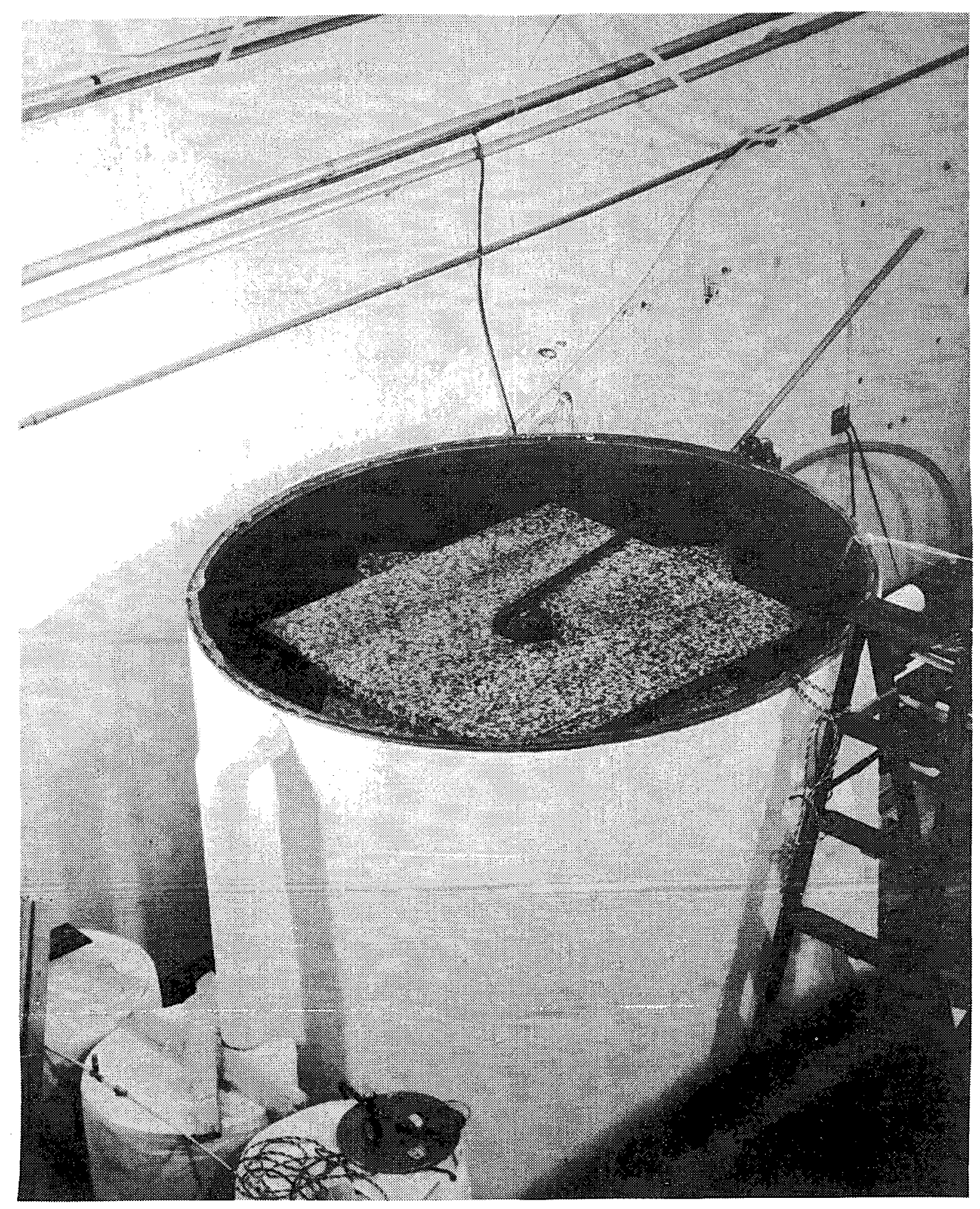

Fig. 18.

Stack in tank. 
5. Place flex hose on fill pipe number 8 (see RT122D001A). Run the other end of the flex hose into the 55-gallon drum of oil.

6. Turn on pump. It should take approximately 5 minutes to drain the 55-gallon drum into the tank.

7. Turn off pump. Get the next drum of oil and repeat steps 5-7 until oil is about a foot above the granite stack.

To operate (circulate oil through tank), use the following procedure.

1. Turn off pump.

2. Cap off fill pipe.

3. Close V8 and V9.

4. Open V1 and/or V2; open V2 for heat exchanger.

5. Open V3 or V4 and V5 for filter.

6. Open V12, V7, V6, V10, and V11.

7. Turn on pump to circulate fluid.

The float switch will control the 3-way valve labeled 2 in RT122D001A to either recirculate from the main tank through $V 6$ and $V 7$ or pull oil from the expansion tanks through V12.

The procedure for draining oil is as follows.

1. Turn off pump.

2. Close V10 and V11.

3. Open V1.

4. Open V6 and V7; close V12 if draining froin main tank. The 3-way valve must be set for recycle.

5. Open V12; close V6 and V7. If draining from expansion tank, the 3-way valve must be set for expansion tank.

6. Open V3 or V4 and V5 for filter.

7. Close V9 and open V8.

8. Connect flex hose from fill pipe to empty 55-gallon drum. 
9. Start pump and fill 55-gallon drum.

10. Stop pump and get another drum. Repeat steps 8-10 until tanks are drained.

\subsection{TFT Probe Mockup Design}

The TFT described in Ref. 1 was used at temperatures below $90^{\circ} \mathrm{C}\left(194^{\circ} \mathrm{F}\right)$. The temperature probe was redesigned for this experiment to withstand temperatures of up to $250^{\circ} \mathrm{C}\left(482^{\circ} \mathrm{F}\right)$. In addition, a second bare detector was added to facilitate the data analysis. In the present design, the gadolinium-filtered and one of the bare detectors are located at equal distances at either side of the neutron source. The new bare detector is located farther from the source than the first two, but at the same distance from the source as the cadmium-filtered detector. Its response would be used in conjunction with that of the other bare detector to correct the $\mathrm{Cd}$ detector counts for the difference in spacing. The original bare detector and Gd-filtered detector are closer to the source than the $\mathrm{Cd}$ detector. To properly compare absolute counting rates, a correction must be applied to normalize the far detector counts to the near detector counts. The ratio of far to near bare detectors would provide this correction factor.

The source shielding material was replaced by a high melting point nylon (Zytel 101). The nylon has a hydrogen density nearly equal to that of water, making it a good moderator for the source neutrons. The thermalized neutrons will be captured by $\mathrm{Cd}$ disks placed every inch along the cylindrical shield and by the $\mathrm{Cd}$ cylinder surrounding the shield.

High-temperature coax is used for the connections to the detectors. The probe is housed in anoil-tight casing.Thermocouple wires monitor the temperature of the detectors in the probe so that corrections described in Section 5 can be applied.

The far bare detector was a 10 -inch-long ${ }^{3} \mathrm{He}$ detector. The far Cd-covered, near bare, and near Gd-covered detectors were 4-inch ${ }^{3}$ He detectors. The 10-inch detector was used because another 4 -inch detector was not available. The response of a 10-inch detector compared to a 4 -inch one at the same distance was estimated based on other experiments at IRT.

The near detectors were centered at 8 inches from the source. The far 4 -inch detector was $16-1 / 2$ inches from the source and the 10-inch detector was 20 inches from the source. The source strength was 3.2 micrograms. Each detector had $c$. thermocouple strapped to it to monitor its temperature.

Figure 19 shows the probe in relation to the granite stack and test stand. Note that the horizontal and vertical scales are different. 


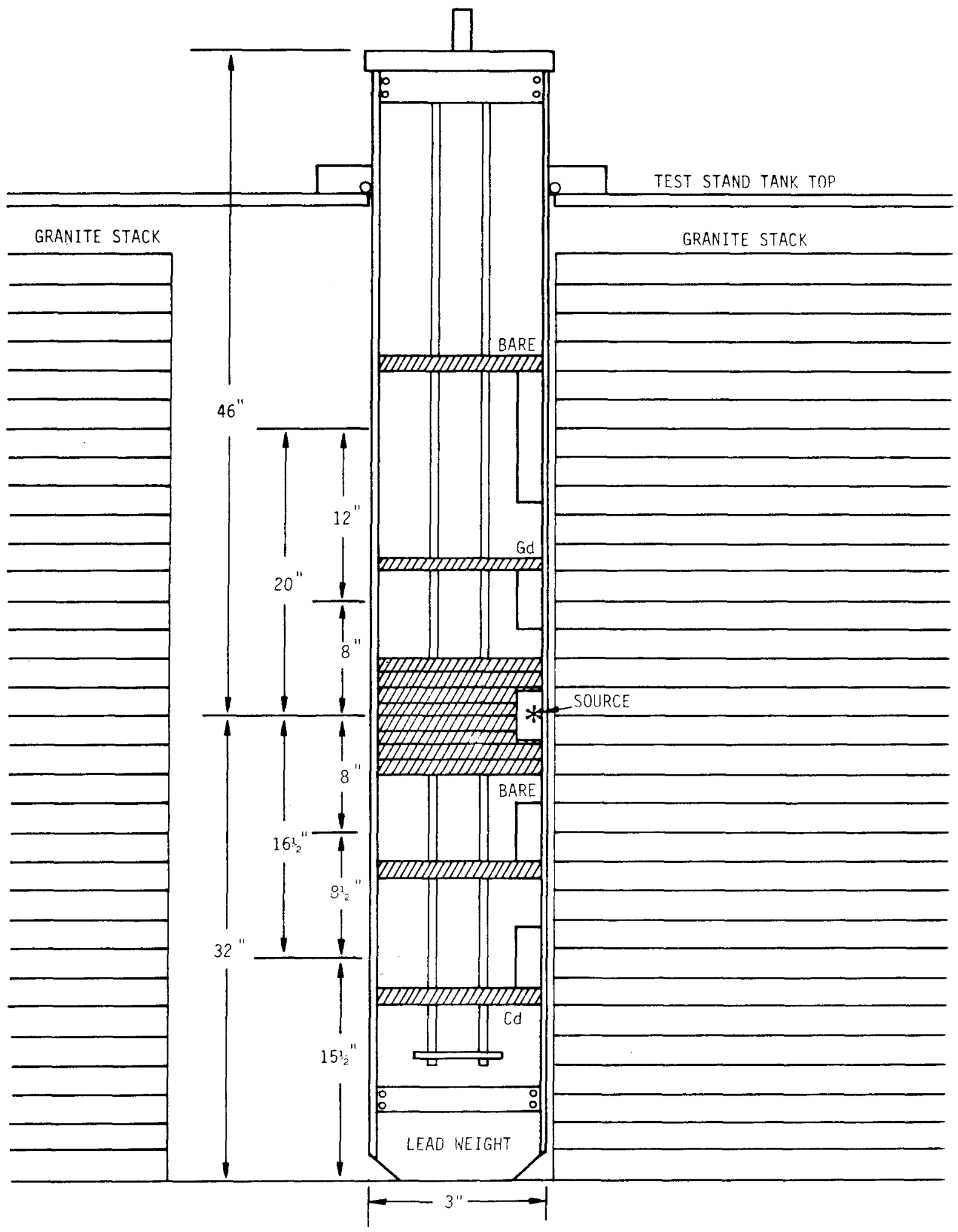

Fig. 19.

True formation temperature probe mockup in test stand. The vertical and horizontal scales are different. 
The source-to-detector distance of 8 inches was chosen for two reasons. The major reason was space. The near detectors were placed as close as possible to the source so that the far detectors could also be placed as close as possible in order to minimize end effects from the top of the granite stack and bottom of the granite stack. The source-to-detector distance also gives the highest count rate possible for the experiment.

The degree of thermalization at this distance can be estimated as 70 to $80 \%$ complete from experiments performed at IRT. In these experiments the ratio of bare detector counts to $\mathrm{Cd}$-covered detector counts was measured as the source-to-detector distance was varied. The ratio was constant at distances greater than approximately 12 inches, indicating complete thermalization.

Complete thermalization is not required for this probe, however, because it measures changes in thermalization with temperature, moderating power and absorption. Any nonthermalization will be accounted for when calibrating the probe.

\section{TFTS DATA ANALYSIS METHODS}

In order to determine the temperature of a given formation, the counts due to thermal neutrons from each detector must be calculated for comparison with the calibration cross plot(s). When the temperature of the formation is low and the moderating power of the medium is high, the epithermal contribution to the total counts can be estimated from the total counts from the cadmium-filtered detector as discussed in Section 2.2. Subtracting the epithermal counts from the total gives the thermal portion of the counts.

When the temperature of the medium is high and moderating power low, the thermal spectrum given by Eq. (1) in Section 2.1 affects the cadmium detector count rate. In addition, the constant $C$ in Eq. (1) becomes large. The correction to the count rate due to epithermal neutrons that have not yet slowed down to the thermal region is large.

The following subsections describe a technique that can be used to calculate the thermal and epithermal portions of the count rates for each detector accurately. The variation of this technique used to analyze the present data is also described in this section.

\subsection{Epithermal Integrals}

The relative counts due to epithermal neutrons are given by the following equation [refer to Eqs. (10) and (11)], 


$$
\frac{C^{e p}}{C}=\int_{E_{o}}^{E_{\max }} d E \cdot E^{-1} \cdot \epsilon(E) \cdot \tau_{f}\left(E, t_{f}\right)
$$

$\epsilon(E)$ is the detector efficiency at energy $E . \tau_{f}\left(E, t_{f}\right)$ is the filter transmission at energy $E$ for a filter of thickness $t_{f} . E_{o}$ is a cutoff energy for the $1 / E$ slowing-down spectrum usually taken to be between $0.1 \mathrm{eV}$ and $0.2 \mathrm{eV}$ for a uniformly distributed source in an infinite medium. ${ }^{2}$

This integral Eq. (14) was evaluated numerically by computer for each detector. It is independent of formation properties. The efficiency $\epsilon(E)$ for a bare ${ }^{3}$ He detector was obtained from a calculated curve verified by experiment. The curve was parameterized resulting in the following equation for $\epsilon(E)$.

$$
\begin{aligned}
\frac{\epsilon(E)}{E}= & A_{0}+A_{1} / E+A_{2} / E^{2}+A_{3} / E^{3}+A_{4} / E^{4} \\
& 0.15 e V \leqslant E \leqslant 10 e V,
\end{aligned}
$$

where $A_{0}=-0.012246$

$A_{1}=+0.206951$

$A_{2}=+0.0982504$

$A_{3}=-0.0142303$

$A_{4}=+8.21427 \times 10^{-4}$.

The filter transmission can be calculated from the known neutron cross sections for the filter material and the filter thickness. The transmission is given by:

$$
\tau_{f}\left(E, t_{f}\right)=e^{-N \sigma_{a} t_{f}}
$$

where $\mathrm{N}$ is the number of nuclei per $\mathrm{cm}^{3} \cdot \sigma_{a}$ is the microscopic absorption cross section of the filter for neutrons of energy $E$, i.e., $N \sigma_{a}=\Sigma_{a} \cdot t_{f}$ is the filter thickness.

The transmission for the bare detector is 1 . The transmission of the Gd-filtered detector multiplied by detector efficiency is:

$$
\begin{aligned}
& \frac{\epsilon(E) \bullet \tau_{G d}\left(E, t_{G d}\right)}{E}=A_{0}+\sum_{i=1}^{4} A_{i} / E^{i} \\
& {[0.15 \mathrm{eV} \leqslant E \leqslant 10 \mathrm{eV}] ; \quad{ }^{t_{G d}}=0.002 \text { inches, }}
\end{aligned}
$$


where $\quad A_{0}=-5.13219 \times 10^{-3}$

$$
\begin{aligned}
& A_{1}=+0.174887 \\
& A_{2}=+0.139699 \\
& A_{3}=-0.0324042 \\
& A_{4}=+1.74754 \times 10^{-3} .
\end{aligned}
$$

For the Cd-covered detector we have:

$$
\begin{aligned}
& \frac{\epsilon(E) \cdot \tau_{C d}\left(E, t_{G d}\right)}{E}=A_{0}+\sum_{i=1}^{6} A_{i} / E^{i} \\
& {[0.15 \mathrm{eV} \leqslant E \leqslant 10 \mathrm{eV}], \quad{ }^{t}{ }_{C d}=0.03 \text { inch, }}
\end{aligned}
$$

where $\quad A_{0}=-5.71921 \times 10^{-3}$

$$
\begin{aligned}
& A_{1}=+0.132283 \\
& A_{2}=+0.287876 \\
& A_{3}=-0.144048 \\
& A_{4}=-1.80898 \times 10^{-3} \\
& A_{5}=+7.01927 \times 10^{-3} \\
& A_{6}=-6.81742 \times 10^{-4} .
\end{aligned}
$$

The integrals of Eq. 14 were evaluated numerically by splitting the energy range $0.15 \mathrm{eV} \leqslant \mathrm{E} \leqslant 10 \mathrm{eV}$ into 5000 steps and using the polynomial approximations to calculate the function at each energy. The results for a cutoff energy of $0.15 \mathrm{eV}$ are

$$
\begin{array}{lll}
\mathrm{E}_{\mathrm{o}}=0.15 \mathrm{eV} & \mathrm{I}_{\mathrm{b}}=0.87143 & \text { Bare } \\
& { }^{\mathrm{I}} \mathrm{Gd}=0.82878 & \text { Gadolium filtered } \\
\mathrm{I}_{\mathrm{Cd}}=0.67787 & \text { Cadmium filtered } .
\end{array}
$$

For a cutoff energy of $0.4 \mathrm{eV}$ (epicadmium region only), the integrals are

$$
\begin{aligned}
& E_{0}=0.4 \mathrm{eV} \mathrm{I}_{\mathrm{b}}=0.74515 \\
& \mathrm{I}_{\mathrm{Gd}}=0.75776 \\
& \mathrm{I}_{\mathrm{Cd}}=0.65848 .
\end{aligned}
$$




\subsection{Evaluation of Thermal Integrals}

The thermal integrals depend on the parameter $\mathrm{kT}_{n}$. These integrals were calculated using 100 steps in the energy intervals as shown below for the bare, gadolinium-filtered, and cadmium-filtered detectors, respectively.

$$
\begin{aligned}
& I_{4}=\int_{0}^{0.5 e V} d E \epsilon(E) \cdot \exp \left(-E / k t_{n}\right) \cdot\left(k T_{n}\right)^{-3 / 2} \\
& I_{5}=\int_{005}^{0.5 \mathrm{eV} d E \epsilon(E) \cdot \tau_{G d}\left(E, t_{G d}\right) \cdot \exp \left(-E / k T_{n}\right)\left(k T_{n}\right)^{-3 / 2}} \\
& I_{6}=\int_{0.3}^{0.5 \mathrm{eV}} \mathrm{dE} \epsilon(E) \cdot \tau_{C d}\left(E, t_{C d}\right) \cdot \exp \left(-E / k T_{n}\right)\left(k T_{n}\right)^{-3 / 2}
\end{aligned}
$$

Below $0.005 \mathrm{eV}$, the transmission through the Gd filter is essentially zero. Below $0.3 \mathrm{eV}$, the transmission through the $\mathrm{Cd}$ filter is zero. Above $0.5 \mathrm{eV}$, the exponential term is very small and the efficiency and transmission factors are less than $1 \%$.

The integrals were calculated for $\mathrm{kT}_{n}=0.0200$ to $0.0950 \mathrm{eV}$ in steps of 0.0001 and stored in a disk file. Whenever $k T_{n}$ is changed in the computer code, the file is accessed to obtain the new integral. This saves considerable time in the evaluation of the thermal integrals. The functions $\epsilon(E) \bullet \tau_{f}\left(E, t_{f}\right)$ are approximated by the polynomials given in Table II.

\subsection{Computer Code}

Trial values for $\mathrm{N}, \mathrm{kT}_{\mathrm{n}}$, and $\mathrm{C}$ are input to the computer. The counts are calculated and compared to the experimental counts. Chi-squared $\left(\chi^{2}\right)$ is used as a measure of how close $\mathrm{N}, \mathrm{kT}_{\mathrm{n}}$, and $\mathrm{C}$ are to the true values. Chi-squared is defined as follows:

$$
\chi^{2}=\sum_{i=1}^{3}\left(c_{i}^{\text {calc }}-c_{i}^{\exp }\right)^{2} / \sigma_{i}^{2},
$$


TABLE II

APPROXIMATIONS TO $\epsilon(E) \bullet \tau_{f}\left(E, t_{f}\right)$

Bare Detector

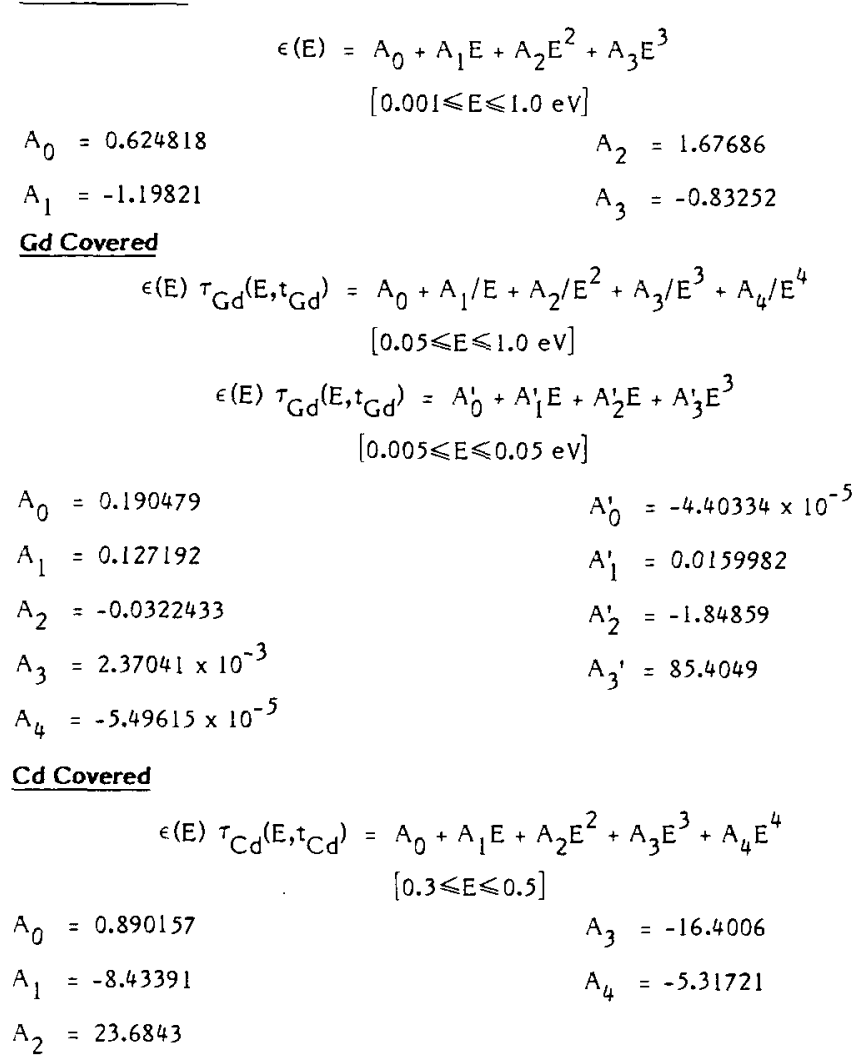

where $C_{i}^{\text {calc }}$ are the calculated counts, $C_{i}^{\text {exp }}$ are the experimental counts, and $\sigma_{i}$ are the uncertainties in the experimental counts. $\mathrm{i}=1,2,3$ for bare, Gd-covered, and Cd-covered detectors.

The program attempts to reduce $\chi^{2}$ by finding the change (derivative) of $\chi^{2}$ with a change in each of the parameters $N, k T_{n}$, and $C$. The new values of the parameters are values that produce the largest change in $\chi^{2}$; that is, the program searches for the solution along the gradient of $\chi^{2}$. From the new values of the parameters, new values of $\mathrm{C}_{\mathrm{i}}^{\text {calc }}$ are computed and the process is repeated.

As the solution is approached, the gradient of $\chi^{2}$ becornes very small so that no significant changes occur in the parameters. When this happens, the calculation proceeds to another algorithm to minimize $\chi^{2}$. The new procedure consists of changing each parameter in turn by a fixed step until $\chi^{2}$ begins to increase instead of decrease. The program stops when $\chi^{2}$ becomes less than some minimum value usually set to 1.0 .

With a reasonable guess for the initial values of $N, k T_{n}$, and $C$, the above procedure runs less than 60 seconds. With good starting values the time can be reduced 
to 10 to 20 seconds. The final step is to print out the thermal and epithermal parts of the total counts for each detector. The ratio $R=C_{G d}^{\text {th }} / C_{B}^{\text {th }}$ is also calculated and printed out. $\mathrm{R}$ is correlated with formation temperature and $\mathrm{C}$ is a measure of formation moderating power.

The fitting procedure described above requires the corrected $\mathrm{Cd}$ counts, which would be measured at the same distance as the bare and gadolinium-filtered detectors. The $\mathrm{Cd}$ detector counts obtained at the far distance are multipled by a correction factor to estimate the $\mathrm{Cd}$ near counts. The correction factor is estimated by comparing the counts from a bare far detector, detector number four, to a bare near detector. This must assume that the ratio of two bare detectors will be the same as the ratio of two Cd-covered detectors.

Unfortunately, the only far bare detector available for this initial experiment was a 10-inch detector. Another correction factor is then needed to convert from 10-inch bare counts (far) to 4-inch bare counts (far). This factor is known at low temperature from previous experiments at IRT. Time did not permit measurement of this ratio in the granite formation at high temperatures.

Because of the relatively large uncertainty of these two correction factors and their behavior at high temperature, a variation of the above technique was used to analyze the data from the granite formation. The alternate technique uses the fact that for a given porosity, the slowing-down parameter $\mathrm{C}$ should be independent of temperature. We also assume that for the low porosity in this experiment (2\%), the formation density does not change significantly. On this basis, $\mathrm{C}$ is a constant that can be calculated at low temperature and used for all temperatures. It need not be varied by the computer fitting code. The far-to-near correction factor (called S/D on the computed printouts) can be varied instead. By fitting to S/D, one need not use the fourth detector to try and determine the near Cd-covered counts at all temperatures. The near counts will be the far counts multiplied by the S/D ratio. The program will still come to a least square solution giving $\mathrm{N}, \mathrm{kT}$, and S/D for each temperature. The input data are the counts from the near bare, near Gd-covered, and far $\mathrm{Cd}$-covered detector.

The constant $\mathrm{C}$ was calculated assuming that at the lowest temperature measured $39.4^{\circ} \mathrm{C}\left(103^{\circ} \mathrm{F}\right)$, the $\mathrm{Cd}$ detector was counting no thermal spectrum neutrons. Then $\mathrm{C}_{\mathrm{Cd}}$ $=\mathrm{C}_{\mathrm{Cd}}^{\mathrm{ep}}$, and

$$
C_{c d}^{e p}=C \int_{E_{0}}^{E} d E \cdot E^{-1} \cdot \epsilon(E) \cdot \tau_{f}\left(E, t_{c d}\right)
$$


where $\epsilon(E)$ is detector efficiency and $\tau_{f}$ is the Cd filter transmission. The integral was calculated using the approximation for $\epsilon(E) \cdot \tau_{f}\left(E, t_{c d}\right)$ given in Table I. The result is 0.67787 using 5000 energy steps between $E_{0}=0.15 \mathrm{eV}$ and $E_{\max }=10 \mathrm{eV}$. Thus,

$$
\mathrm{C}=\mathrm{C}_{\mathrm{Cd}}^{\mathrm{ep}} / 0.67787
$$

$\mathrm{C}_{\mathrm{Cd}}^{\text {ep }}$ was measured at the far detector spacing only. To estimate $\mathrm{C}_{\mathrm{Cd}}^{\mathrm{ed}}$ at the near detectors, we apply a correction factor for distance from the source. We have measurements of counts from two bare detectors. One is 4 inches long at the near position. One is 10 inches long at the far position. Because the $\mathrm{Cd}$-covered detector is at the far position, we need to convert 10-inch detector counts to 4-inch detector counts at the far position. This conversion ratio $R$ was measured previously at IRT for a low-porosity low-temperature formation. It varied between $R=3.10$ and $R=3.33$, depending on distance from the source. For the present analysis, we will use a ratio of 3.27. C can now be calculated as follows.

$$
\begin{aligned}
& \text { Data at } 39.4^{\circ} \mathrm{C}\left(103^{\circ} \mathrm{F}\right) \\
& \text { Counts: } \mathrm{C}_{\mathrm{B}} \text { (near) }=576027 \quad \text { (4-inch detector) } \\
& \mathrm{C}_{\mathrm{Cd}} \text { (far) }=20681 \\
& \mathrm{C}_{\mathrm{B}}(\mathrm{far})=122099 \quad(10 \text {-inch detector) } .
\end{aligned}
$$

Using $R=3.27$, we find

$$
\mathrm{C}_{\mathrm{B}} \text { (far) } 37339 \text { (4-inch detector) . }
$$

The ratio of near/far S/D is then

$$
\frac{C_{B} \text { near }}{C_{B} \text { far }}=\frac{576027}{37339}=15.43 .
$$

Applying this ratio to the $\mathrm{Cd}$ detector, we estimate

$$
C_{c d}^{\text {ep }} \text { (near) }=319043 \text { (4-inch detector). }
$$

Then $C=319043 / 0.67787=47.066 \times 10^{4}$.

In the fitting program, the input $C$ is multiplied by $10^{4}$ so the input value for $C$ is 47.066 . 


\subsection{Analysis Results}

The spacers between the granite stack gave the formation a fracture porosity of approximately $2 \%$. After the test stand was checked out mechanically, data collection was started. The formation temperature was $52.8^{\circ} \mathrm{C}\left(127^{\circ} \mathrm{F}\right)$ when the experiment began (it had not cooled down from the testing phase). The temperature probe mockup was side walled with the neutron source and detectors next to the formation. Detector pulses were counted for several 60 -second time periods to check reproducibility. Then 4-minute counting periods were used throughout the experiment.

The probe was also turned $180^{\circ}$ into the borehole containing the oil. The high porosity of the borehole was evident in the decrease in the $\mathrm{Cd}$-covered detector counts. The low-porosity Cd count was $95 \mathrm{cps}$. The high-porosity count was $41 \mathrm{cps}$.

The experiment proceeded by increasing the formation temperature in steps of 20 to $25^{\circ} \mathrm{F}$ and stopping to collect data. Approximately one day was needed for each temperature stop. Figure 20 is a plot of the formation temperature on various dates through a 2-week period. The temperature of the probe and borehole was usually 2 to $5^{\circ}$ higher than the formation except when the test stand was allowed to stabilize over a weekend.

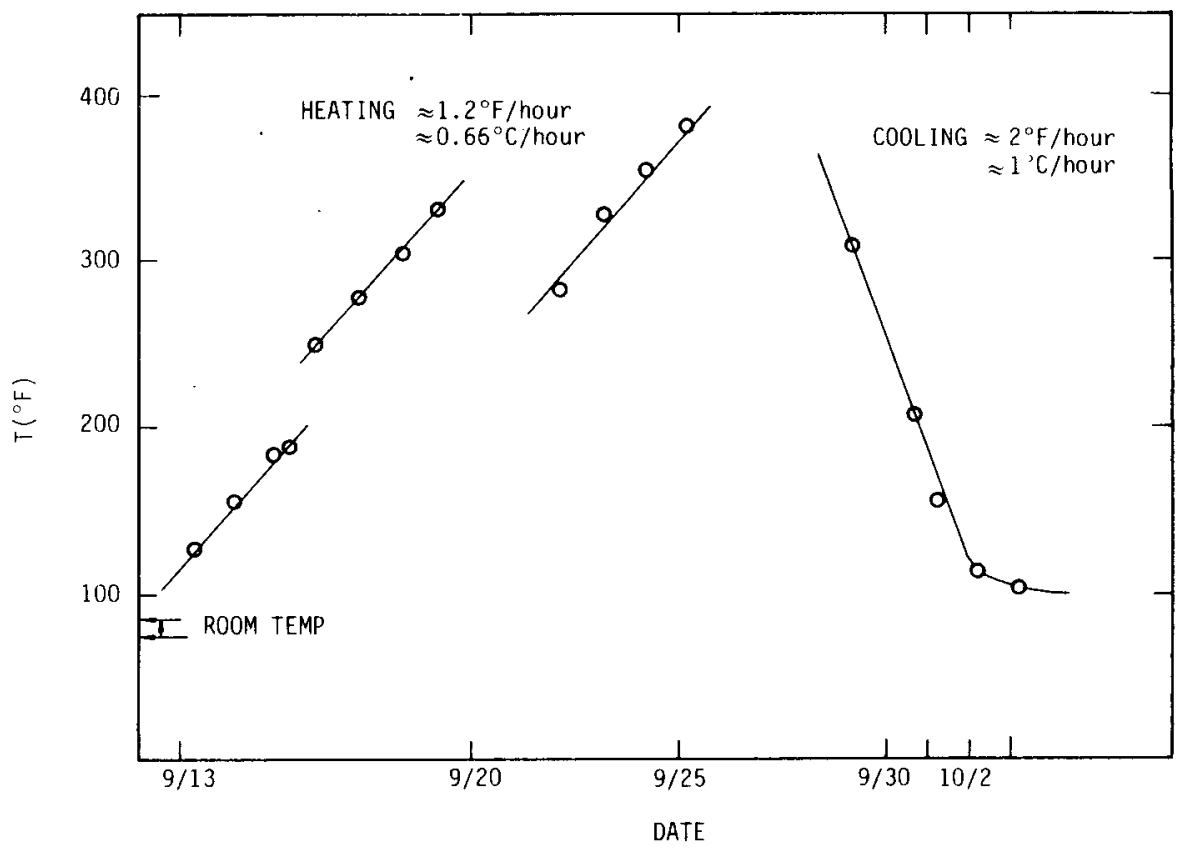

Fig. 20.

Geothermal test stand temperature versus time.

The response of the ${ }^{3} \mathrm{He}$ detectors versus temperature (Ref. 9) is discussed in the Appendix $A$ to this report. The results presented there were used to correct the raw 
detector counts for ambient temperature effects. The corrected counts are obtained from raw counts by multiplying by a correction factor $T_{R}$ found to be related to temperature $\mathrm{T}$ by the equation

$$
T_{R}=1.004956-0.4450819 \times 10^{-4} \mathrm{~T}
$$

where $T$ is in degrees $F$.

Table III gives the corrected detector counts at various formation temperatures. The thermal counts were then calculated using the second technique discussed in Section 4.3. The cutoff energy $E_{0}$ for the slowing-down spectrum in Eqs. (10) and (11) was taken as $0.15 \mathrm{eV}$. The value use for the slowing-down spectrum constant $\mathrm{C}$ was 47.

TABLE III

TOTAL COUNTS CORRECTED FOR TEMPERATURE OF DETECTORS

\begin{tabular}{|c|c|c|c|c|c|c|c|}
\hline $\mathrm{T}^{\mathrm{O}} \mathrm{F}$ & \multicolumn{2}{|c|}{$\begin{array}{c}\text { FAR } \\
\text { 10" BARE }\end{array}$} & $\begin{array}{c}\text { NEAR } \\
\text { GADOLINIUM }\end{array}$ & $\begin{array}{l}\text { NEAR } \\
\text { 4" BARE }\end{array}$ & \multicolumn{3}{|c|}{$\begin{array}{c}\text { FAR } \\
\text { CADMIUN }\end{array}$} \\
\hline 103 & $122099 \pm$ & $\pm \quad 283$ & $398846 \pm 1895$ & $576027 \pm 618$ & 20681 & & 371 \\
\hline 114 & $122018 \pm$ & \pm 230 & $393100 \pm 1028$ & $575376 \pm 470$ & 20551 & \pm & 152 \\
\hline 127 & $118016 \pm$ & \pm 664 & $398736 \div 3584$ & $572868 \pm 2124$ & 22744 & \pm & 388 \\
\hline 152 & $121804 \pm$ & $\pm \quad 484$ & $404780 \pm 936$ & $581348 \pm 1280$ & 24608 & \pm & 536 \\
\hline 165 & $128169 \pm$ & $\pm \quad 458$ & $411701 \pm 1784$ & $588278 \pm 529$ & 21490 & \pm & 45 \\
\hline 182 & $126080 \pm$ & $\pm \quad 495$ & $409291 \pm 2095$ & $584618 \pm 467$ & 23596 & \pm & 364 \\
\hline 187 & $127830 \pm$ & $\pm \quad 33$ & $411318 \pm 1801$ & $584646 \pm 1221$ & 27110 & 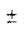 & 2895 \\
\hline 206 & $130787 \pm$ & \pm 380 & $407645 \pm 1434$ & $597939 \pm 972$ & 22346 & \pm & 137 \\
\hline 248 & $140165 \pm$ & \pm 241 & $430891 \pm 1902$ & $597991 \pm 391$ & 23813 & \pm & 54 \\
\hline 278 & $149301 \pm$ & \pm 4726 & $434654 \pm 2920$ & $605392 \pm 593$ & 25040 & \pm & 300 \\
\hline 282 & $145794 \pm$ & $\pm \quad 432$ & $444120 \pm 1824$ & $609276 \pm 570$ & 24733 & \pm & 183 \\
\hline 302 & $150032+$ & $\pm \quad 562$ & $435508 \pm 659$ & $616189 \pm 1034$ & 25601 & \pm & 52 \\
\hline 307 & $146252 \pm$ & \pm 139 & $450461 \pm 1530$ & $610695 \pm 1331$ & 24474 & \pm & 91 \\
\hline 322 & $154617 \pm$ & $\pm \quad 518$ & $458929 \pm 1012$ & $619184 \pm 314$ & 25901 & \pm & 116 \\
\hline 327 & $156184 \pm$ & $\pm \quad 686$ & $454275 \pm 744$ & $620313 \pm 232$ & 26134 & 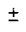 & 66 \\
\hline 357 & $158180 \pm$ & $\pm \quad 516$ & $468017 \pm 1717$ & $622965 \pm 1205$ & 26100 & \pm & 270 \\
\hline 380 & $160885 \pm$ & $\pm \quad 559$ & $472426 \pm 728$ & $626348 \pm 972$ & 26172 & \pm & 187 \\
\hline
\end{tabular}

The result of the computer analysis is given in Table IV. The fitting parameters $\mathrm{kT}_{\mathrm{n}}, \mathrm{N}$, and $\mathrm{S} / \mathrm{D}$ are listed as well as the calculated counts from the thermal part of the neutron spectrum. The ratio $\mathrm{C}_{\mathrm{Gd}}^{\text {th }} / \mathrm{C}_{\mathrm{B}}^{\text {th }}$ is the indicator of formation temperature at constant porosity and formation neutron absorption cross section used in the lowtemperature experiments with the TFTS. Figure 21 shows this ratio plotted versus temperature. The data at $\mathrm{T}=114^{\circ} \mathrm{F}, 206^{\circ} \mathrm{F}$, and $302^{\circ} \mathrm{F}$ were excluded as suspect because excessive detector noise was occasionally seen during the experiment, causing erroneous results. These points are many sigma away from the line and are assumed to be caused by the noise source.

As with the low-temperature experiments (Ref. 9), the ratio rises linearly with formation temperature giving a good correlation. A weighted least squares fit to the data results in the following equation for $R=C_{G d}^{\text {th }} / C_{B}^{\text {th }}$. 
TABLE IV

RESULTS OF COMPUTER ANALYSIS FOR $\mathrm{E}_{\mathrm{o}}=0.15 \mathrm{eV} . \mathrm{C}=47$

\begin{tabular}{|c|c|c|c|c|c|c|c|}
\hline $\mathrm{T}^{\circ} \mathrm{F}$ & $\mathrm{KT}_{\mathrm{n}}$ & $N$ & $S / D$ & $C_{B}^{\text {th }}$ & $\mathrm{C}_{\mathrm{Gd}}^{\text {th }}$ & $\mathrm{C}_{\mathrm{Cd}}^{\mathrm{th}}$ & $\mathrm{C}_{\mathrm{Gd}}^{\text {th }} / \mathrm{C}_{\mathrm{B}}^{\mathrm{th}}$ \\
\hline 103 & 0.0244 & 187.38 & 15.41 & 166799 & 8607 & 0.4 & 0.0516 \\
\hline 127 & 0.0264 & 176.36 & 14.01 & 163001 & 9898 & 1.3 & 0.0672 \\
\hline 152 & 0.0316 & 173.75 & 12.937 & 172101 & 14623 & 9.8 & 0.0850 \\
\hline 165 & 0.0394 & 165.00 & 14.75 & 178150 & 22495 & 68.0 & 0.1262 \\
\hline 182 & 0.0374 & 165.00 & 13.50 & 174635 & 20141 & 44.7 & 0.1153 \\
\hline 187 & 0.0397 & 161.25 & 11.75 & 174603 & 22336 & 71.5 & 0.1280 \\
\hline 248 & 0.0554 & 154.375 & 13.44 & 188471 & 40729 & 648.0 & 0.2161 \\
\hline 278 & 0.0575 & 158.75 & 12.75 & 196248 & 44677 & 818.0 & 0.2276 \\
\hline 282 & 0.0658 & 155.0 & 13.0 & 200118 & 54364 & 1561.0 & 0.2717 \\
\hline 307 & 0.0717 & 152.5 & 13.125 & 201945 & 60810 & 2234.0 & 0.3011 \\
\hline 322 & 0.0758 & 156.47 & 12.41 & 210456 & 67453 & 2862.0 & 0.3205 \\
\hline 327 & 0.0736 & 157.5 & 12.25 & 210118 & 65109 & 2566.0 & 0.3099 \\
\hline 357 & 0.0853 & 154.58 & 12.37 & 214334 & 77146 & 4287.0 & 0.3599 \\
\hline 380 & 0.0896 & 155.0 & 12.375 & 217117 & 82338 & 5058.0 & 0.3792 \\
\hline
\end{tabular}

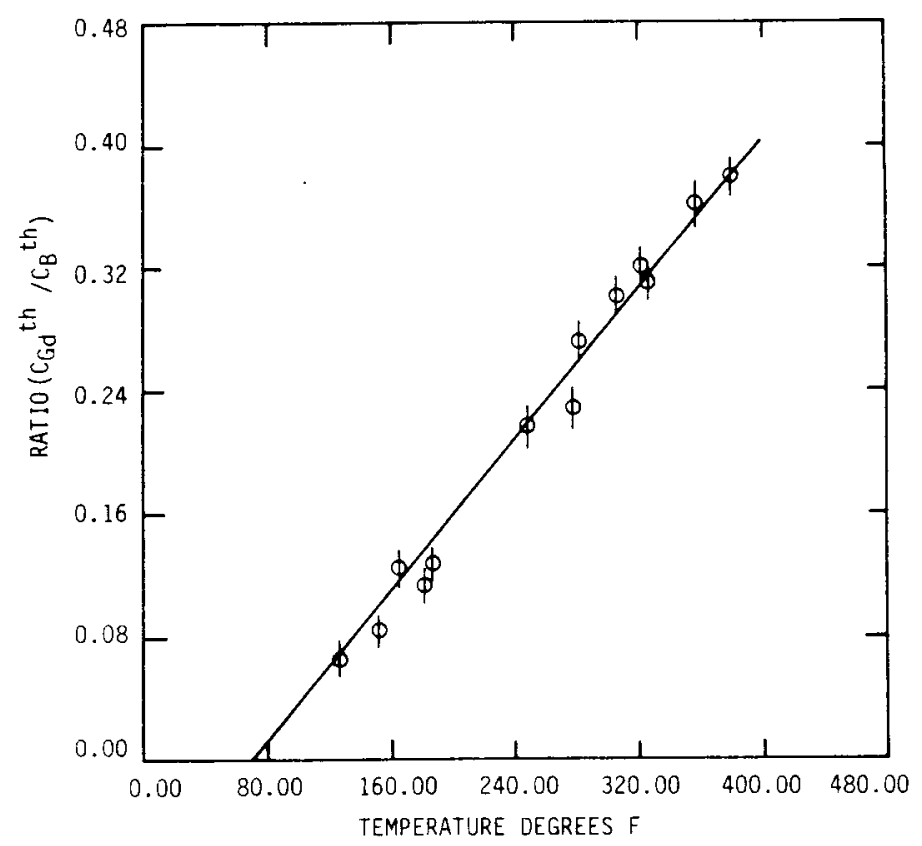

Fig. 21.

Ratio of Gd-covered detector counts to bare detector counts versus temperature.

$$
R=\left(1.222 \times 10^{-3} \pm 3.07 \times 10^{-5}\right) T\left({ }^{\circ} \mathrm{F}\right)-(0.08611 \pm 0.00692)
$$

with a linear correlation coefficient of 0.9936.

\subsection{Summary}

The ratio of thermal neutron counts from the gadolinium-covered detector to thermal neutron counts from the bare detector has been found to be linearly correlated 
with formation temperature at temperatures up to $200^{\circ} \mathrm{C}\left(392.4^{\circ} \mathrm{F}\right)$ in a simulated fractured granite formation with a porosity of about $2 \%$.

The ratio can be determined to high precision by counting longer or by using a larger source. Assuming that the ratio can be determined very precisely, then the uncertainty in temperature obtained from the linear fit can be calculated from the uncertainties in the slope and intercept. The results give a relative uncertainty of approximately $3 \%$ for the present series of measurements. This could be improved by counting longer during calibration of the probe. The ultimate precision depends on the behavior of the tool when varying all three parameters, i.e., neutron absorption, slowing down, and temperature.

Time did not permit experiments to investigate the correlation at other porosities and neutron absorption at high temperature. Previous experiments $1,2,10$ at low temperature indicate that the linear correlation remains throughout a high range of formation porosities and neutron absorption cross sections. The references cited show crossplots of the ratio versus the other two dimensions for temperatures below $100^{\circ} \mathrm{C}$. We have shown in this experiment that the linear relationship between the ratio and formation temperature continues to the high-temperature range. The high-temperature test stand could be used to test the TFTS and verify that the linear correlation exists for porosities and absorption cross sections of interest to temperatures up to $200^{\circ} \mathrm{C}$ $\left(392.4^{\circ} \mathrm{F}\right)$. This could be done by changing the spacing between the granite slabs to vary porosity and adding neutron-absorbing materials in the oil to change the absorption cross section.

The TFTS application with the greatest potential for measuring directly the undisturbed formation temperature is the measurement of the bottomhole temperature during drill bit changes. This region of the borehole should be the least disturbed by the drilling operations. Alternatively, this probe can be used in the logging mode to measure the formation temperature within a volume of radius 6 to 12 inches from the wellbore. This information could be used together with the wellbore temperature to facilitate extrapolation to the undisturbed formation temperature value. Repeated measurements at the same depth could be used as an indication of the rate of formation temperature rebound after the drilling disturbance and/or chilling. The temperature measurements can be performed either in open or cased boreholes.

In addition to the temperature measurement, the TFTS provides simultaneously additional information on the formation porosity, the total capture cross section, and the flashing point depth. The saturated porosity log can be obtained either from the 
cadium-covered detector response or from the ratio of the short-spaced to long-spaced bare detector responses. The porosity logs could be used to detect (locate) fracture zones when the fracture porosity is significantly different from the intergranular porosity of the surrounding formation. The total capture cross section $\Sigma_{a}$ of the formation, can be determined from the cross plots in the same way as the temperature. All other things being equal, the bare detector count rate should be inversely proportional to the total capture cross section of the formation. Finally, as the water changes to vapor in the borehole and/or the formation, the response of the sensors within the probe will change, as discussed in Ref. 6. This can be used to locate the depth at which flashing occurs.

\section{REFERENCES}

1. Vagelatos, Nicholas, Donald K. Steinman, and Joseph John, IRT Corporation, Report No. IRT 7021-019 (March 1978).

2. Meghreblian, R. V. and D. K. Holmes, "Reactor Analysis," McGraw-Hill Book Company, Inc., Chapter 4, p. 69 (1960).

3. Coveyou, R. R., R. R. Bates, and R. K. Osborn, J. Nucl. Energy 2, 153 (1956).

4. Steinman, D. K., D. G. Costello, C. S. Pepper, W. E. Gober, D. B. Breuner, J. S. M. Wilson, J. C. Young, and J. John, ERDA Status Report INTEL-RT 7019-004 (February 1977).

5. Sherman, H., and S. Locke, Society of Petroleum Engineers of AIME, Paper No. SPE 5510 (1975).

6. Vagelatos, Nicholas, D. K. Steinman, and Joseph John, IRT Corporation, Report No. IRT 0062-010 (December 1979).

7. Weber, H. J., C. A. Preskitt, and C. E. Rinehart, "A Cf-252 Thermal Neutron Cross Section Gauge for Interpretation of Neutron Logs for Oil Applications," presented at the Symp. International Sur L'Utilization Du Californium-252, Paris, France (April 1976).

8. Mathews, Mark, "Calibration Models for Fractured Igneous Rock Environments," SPWLA Twenty-First Annual Logging Symposium, Paper L (July 1980).

9. Vagelatos, Nicholas, E. W. Ross, J. M. Dickerson, and Van Nguyen, "Nuclear Logging and Geothermal Log Interpretation: Formation Temperature Sonde Evaluation," Interim Report, IRT 6312-006 (March 1980).

10. Vagelatos, Nicholas, Donald K. Steinman, and Joseph John, "True Formation Temperature Sonde (TFTS)," SPWLA Twentieth Annual Logging Symposium, Paper LL (June 1979). 


\section{APPENDIX A}

\section{RESPONSE OF ${ }^{3}$ He DETECTORS AT HIGH AMBIENT TEMPERATURES}

The response of the ${ }^{3} \mathrm{He}$ detectors used in the TFTS varies slightly with the temperature of the detector. According to the manufacturer, over the temperature range from room temperature to $250^{\circ} \mathrm{C}\left(482^{\circ} \mathrm{F}\right)$ the count rate will change by about $6 \%$.

To correct the data for this variation, the response versus temperature has been measured for the detectors to be used in the TFTS. A linear decrease in the count rate was observed with a fixed thermal neutron spectrum from a moderated ${ }^{252} \mathrm{Cf}$ source. The count rate at $200^{\circ} \mathrm{C}\left(392^{\circ} \mathrm{F}\right)$ changed by less than $2 \%$ relative to the count rate at room temperature.

\section{EXPERIMENTAL ARRANGEMENT}

The detector to be measured was placed between two 500-watt (240 VAC) strip heaters. The heaters were run at $60 \mathrm{VAC}$, however, giving a total for the two heaters of 250 watts. A thermocouple on the surface of the detector was used to measure the detector temperature. Two inches of fiberglass wool insulation were wrapped around the detector and heaters.

The source, 0.6 micrograms of ${ }^{252} \mathrm{Cf}$, was placed in a paraffin moderator to obtain a thermal neutron spectrum. The temperature of the detector was then varied and the response noted. The thermal neutron spectrum remains constant because the source and paraffin remain at room temperature. Figure $A-1$ is a sketch of the experimental arrangement. Figure A-2 shows the electrical connections to the heaters and detector. The temperature controller maintained the detector temperature constant to within $\pm 2^{\circ} \mathrm{F}$.

The lower level discriminator of the SCA was set (at room temperature) to include all counts above the detector noise level. Then the detector high voltage was varied until the plateau region of the detector was reached. The maximum pulse height from the timing amplifier was approximately one volt.

\section{MEASUREMENTS AND RESULTS}

The pulse-height (PH) spectrum was measured with a 256-channel analyzer throughout the temperature range. Figure A-3 shows the $\mathrm{PH}$ spectrum at various temperatures. Significant changes are apparent. The integral of the spectrum (total counts), however, does not vary by more than $2 \%$. The change in the total count rate from room temperature to $200^{\circ} \mathrm{C}\left(392^{\circ} \mathrm{F}\right)$ is plotted in Figure A-4. This figure shows 

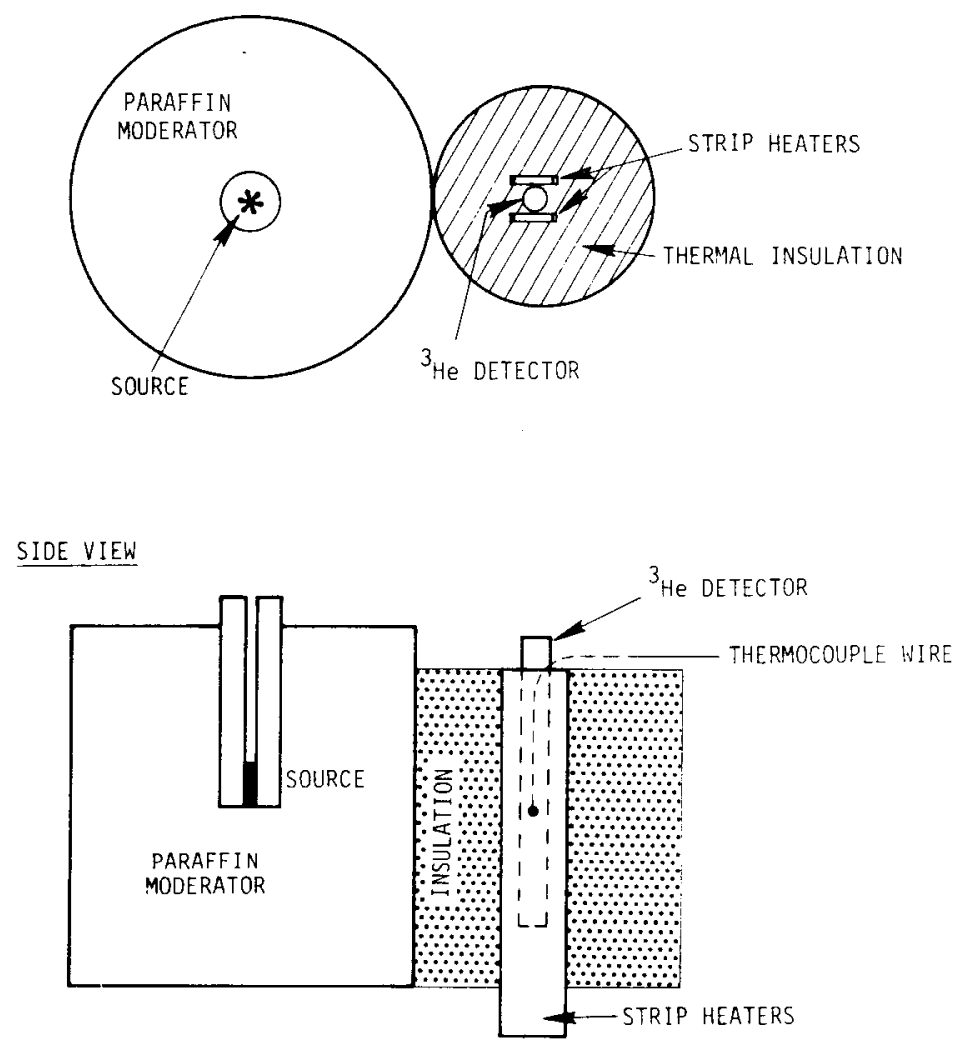

Fig. A-1.

Experimental arrangement for measuring detector response at varying ambient temperature.

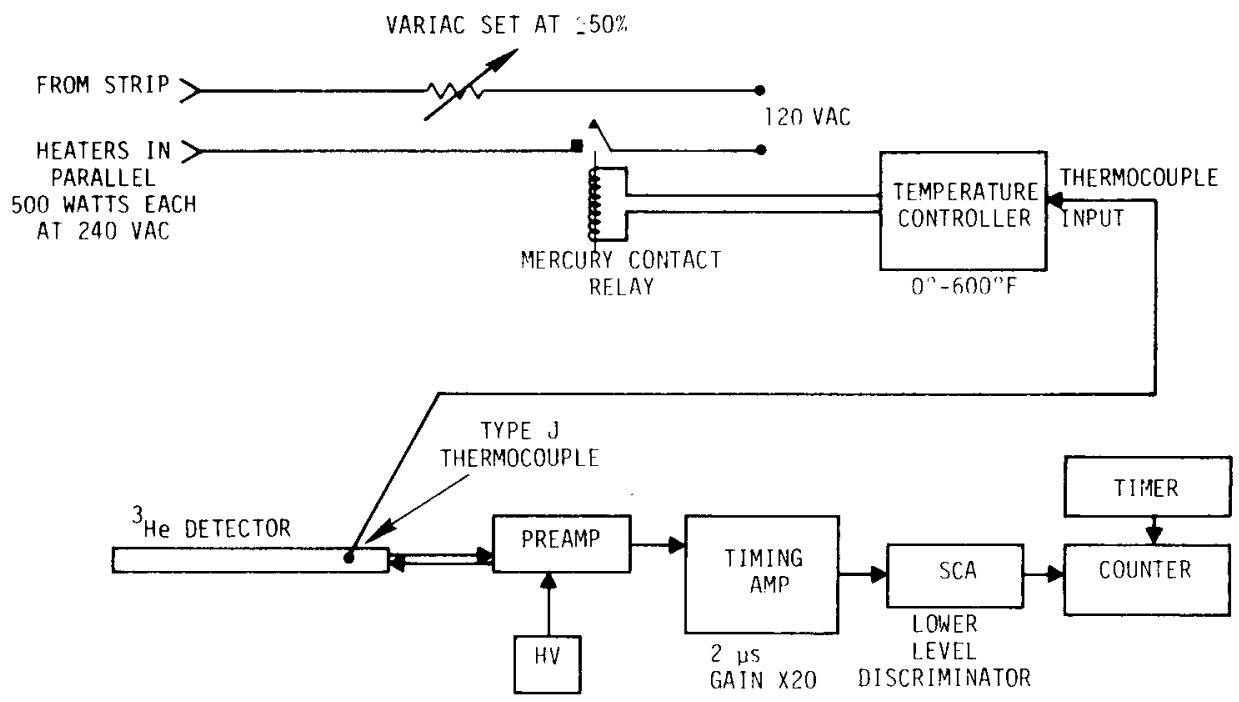

Fig. A-2.

Block diagram of counting electronics and temperature controller for measuring the detector response at varying ambient temperature. 
the results of measurements on two 4 -inch-long ${ }^{3} \mathrm{He}$ detectors. Similar results were obtained for a 10 -inch-long ${ }^{3} \mathrm{He}$ detector. The data can be fit by the line given by

$$
R=1.004956-0.4450819 \times 10^{-4} \cdot \mathrm{T},
$$

where $\mathrm{T}$ is the temperature of the detector in ${ }^{\circ} \mathrm{F}$ and $\mathrm{R}$ is the relative detector response normalized to one at room temperature. This formula will be used to correct the data from the TFTS for the effects of ambient temperature changes in the 4 -inchlong ${ }^{3} \mathrm{He}$ detectors.
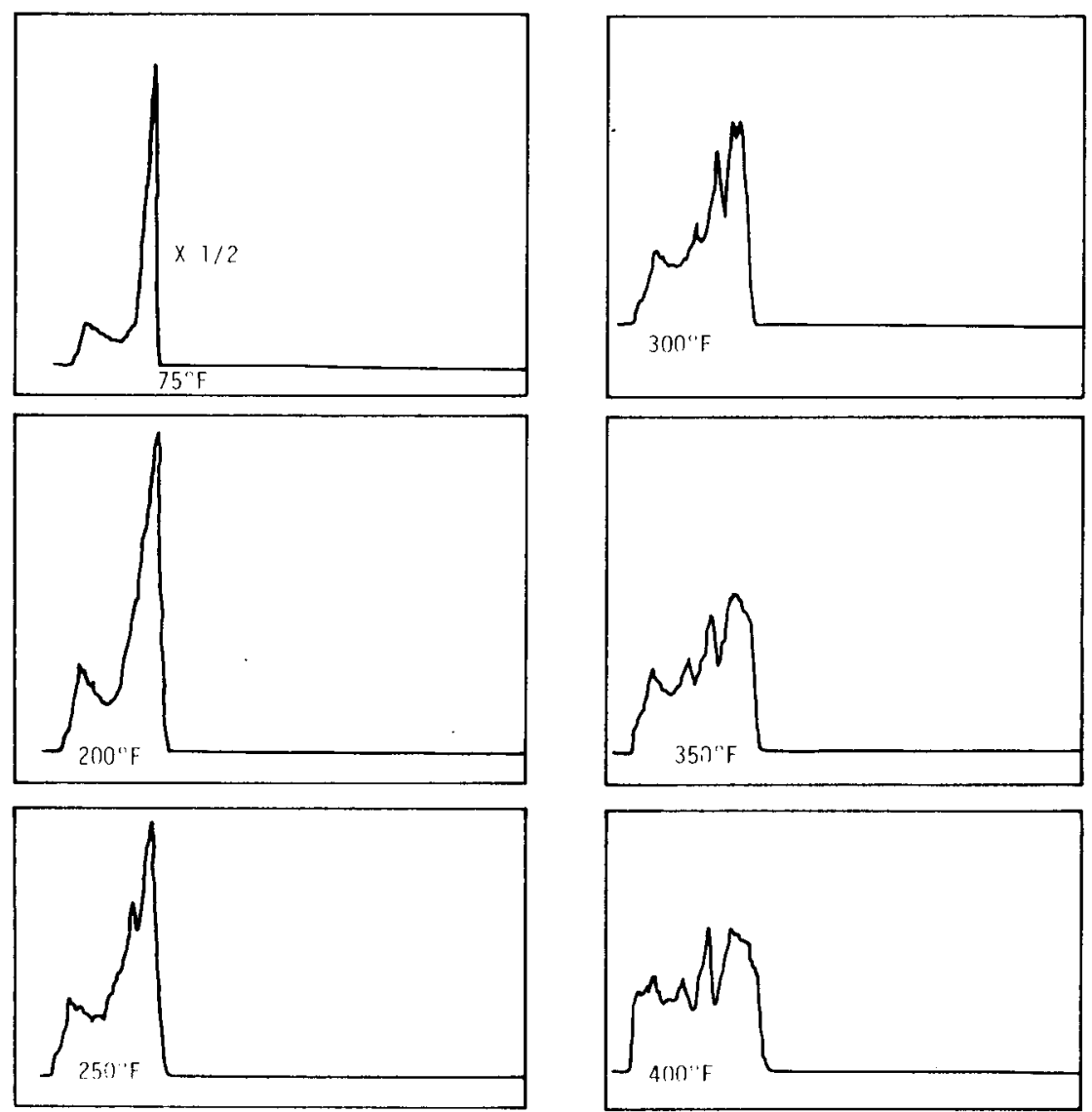
Fig. A-3.
Pulse-height spectra versus temperature for ${ }^{3}$ He detectors. 


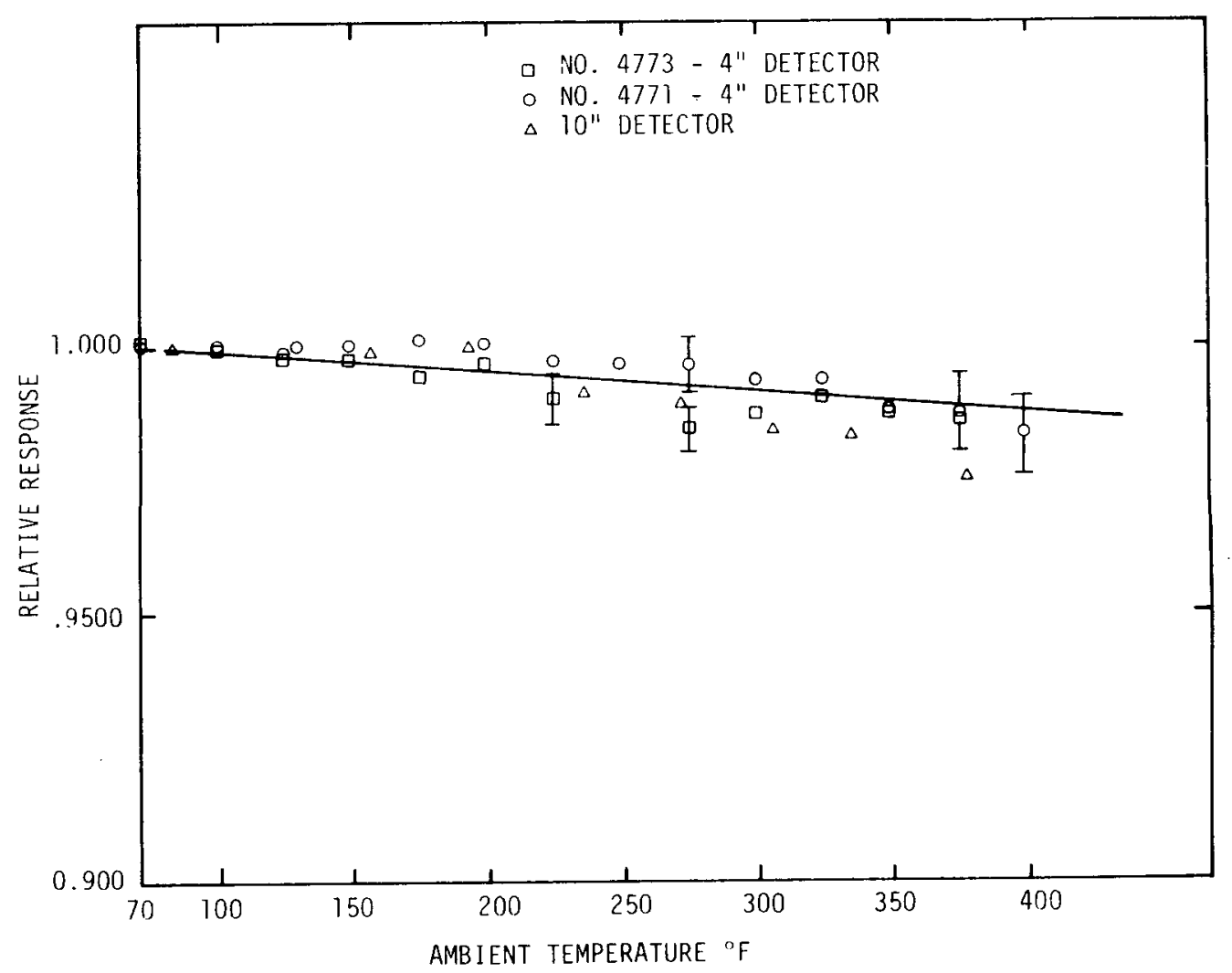

Fig. A-4.

${ }^{3}$ He detector response versus temperature. 


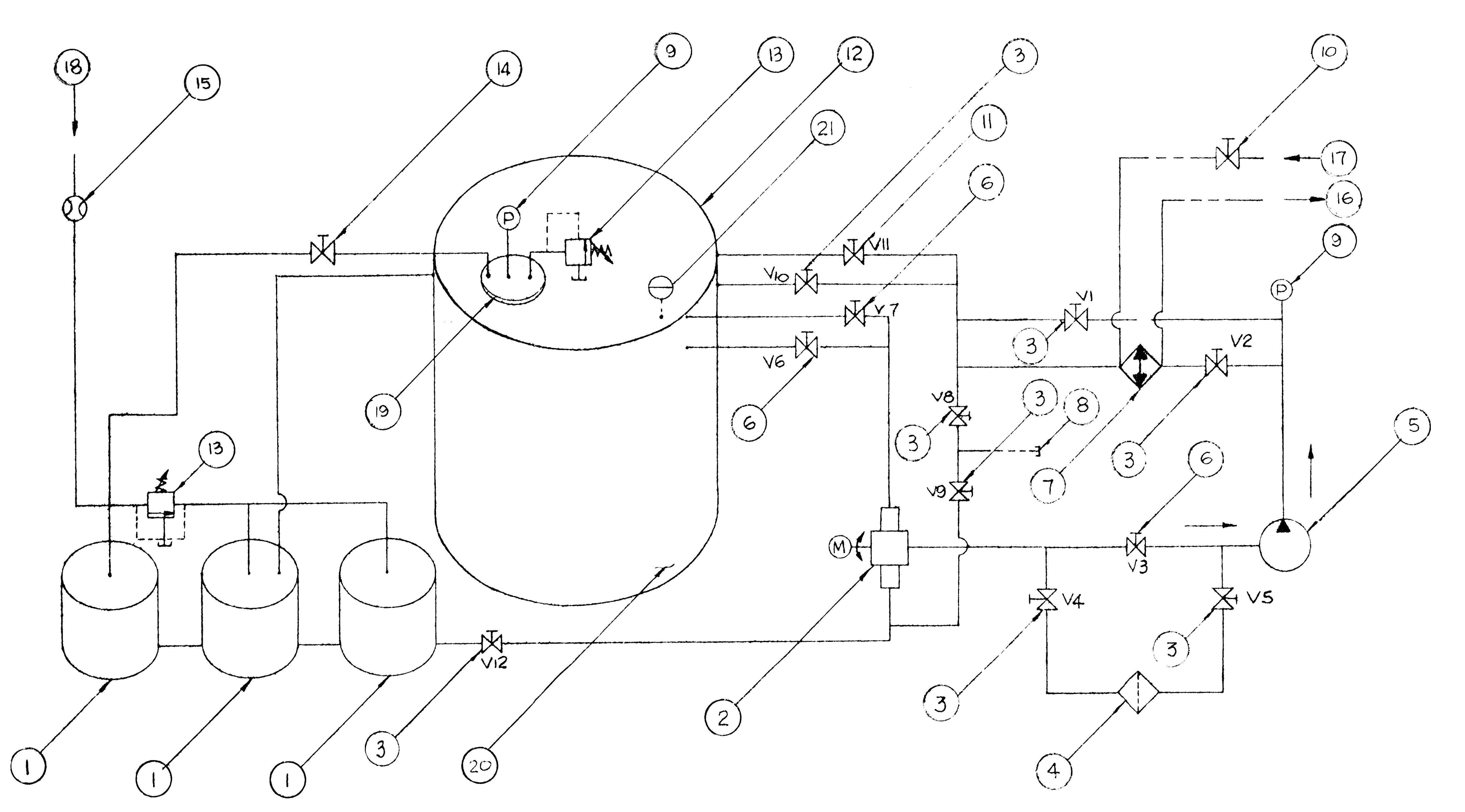

